\title{
Vhl deletion in osteoblasts boosts cellular glycolysis and improves global glucose metabolism
}

\author{
Naomi Dirckx, ${ }^{1}$ Robert J. Tower, ${ }^{1}$ Evi M. Mercken, ${ }^{1}$ Roman Vangoitsenhoven, ${ }^{2}$ Caroline Moreau-Triby, ${ }^{3}$ Tom Breugelmans, ${ }^{1}$ \\ Elena Nefyodova, ${ }^{1}$ Ruben Cardoen, ${ }^{1}$ Chantal Mathieu, ${ }^{2}$ Bart Van der Schueren, ${ }^{2}$ Cyrille B. Confavreux,, 4 \\ Thomas L. Clemens, ${ }^{6,7}$ and Christa Maes ${ }^{1}$ \\ 'Laboratory of Skeletal Cell Biology and Physiology (SCEBP), Skeletal Biology and Engineering Research Center (SBE), KU Leuven, Leuven, Belgium. ${ }^{2}$ Clinical and Experimental Endocrinology, \\ KU Leuven, Leuven, Belgium. ${ }^{3}$ Department of Nuclear Medicine, Hospices Civils de Lyon, Lyon, France. ${ }^{4}$ INSERM UMR1033 - LYOS, Université de Lyon, Lyon, France. ${ }^{5}$ Department of Rheumatology, \\ Hospices Civils de Lyon, Lyon, France. ${ }^{6}$ Department of Orthopaedic Surgery, Johns Hopkins University School of Medicine, Baltimore, Maryland, USA. ${ }^{7}$ Baltimore Veterans Administration \\ Medical Center, Baltimore, Maryland, USA
}

\begin{abstract}
The skeleton has emerged as an important regulator of systemic glucose homeostasis, with osteocalcin and insulin representing prime mediators of the interplay between bone and energy metabolism. However, genetic evidence indicates that osteoblasts can influence global energy metabolism through additional, as yet unknown, mechanisms. Here, we report that constitutive or postnatally induced deletion of the hypoxia signaling pathway component von Hippel-Lindau (VHL) in skeletal osteolineage cells of mice led to high bone mass as well as hypoglycemia and increased glucose tolerance, not accounted for by osteocalcin or insulin. In vitro and in vivo data indicated that Vhl-deficient osteoblasts displayed massively increased glucose uptake and glycolysis associated with upregulated HIF-target gene expression, resembling the Warburg effect that typifies cancer cells. Overall, the glucose consumption by the skeleton was increased in the mutant mice, as revealed by ${ }^{18} \mathrm{~F}-\mathrm{FDC}$ radioactive tracer experiments. Moreover, the glycemia levels correlated inversely with the level of skeletal glucose uptake, and pharmacological treatment with the glycolysis inhibitor dichloroacetate (DCA), which restored glucose metabolism in Vhl-deficient osteogenic cells in vitro, prevented the development of the systemic metabolic phenotype in the mutant mice. Altogether, these findings reveal a novel link between cellular glucose metabolism in osteoblasts and wholebody glucose homeostasis, controlled by local hypoxia signaling in the skeleton.
\end{abstract}

\section{Introduction}

The skeleton serves to provide structural support to the body, allow movement, protect internal organs, regulate calcium homeostasis, and provide niches for hematopoiesis. Additionally, over the past decade the skeleton has emerged as an important endocrine organ, with osteoblast-derived osteocalcin representing a major player in the regulation of glucose and insulin homeostasis $(1,2)$. Osteocalcin, in its undercarboxylated form, is released as a hormone into the circulation and promotes pancreatic $\beta$ cell proliferation, insulin production, and peripheral insulin sensitivity (3). Osteoblasts in turn respond to insulin signaling by increasing bone remodeling and the production and bioavailability of undercarboxylated osteocalcin $(4,5)$.

Recent reports also implicated osteocalcin-independent mechanisms in the bone-metabolism interplay. For example, genetically induced ablation of osteoblasts or conditional inactivation of glycogen synthase kinase- $3 \beta$ or $\beta$-catenin in osteoblasts led to systemic metabolic alterations, which could not be fully rescued or explained by congruent changes in serum osteocalcin (6-8). These studies indicated that osteoblasts can additionally

Conflict of interest: The authors have declared that no conflict of interest exists Submitted: October 23, 2017; Accepted: December 28, 2017. Reference information: / Clin Invest. 2018;128(3):1087-1105. https://doi.org/10.1172/JCI97794. influence global glucose homeostasis and energy metabolism through mechanisms that are as yet unknown and that may, or may not, be endocrine in nature.

Hypoxia-driven pathways play major roles in pathological conditions such as cancer and metastasis, but are also vital in normal development and tissue homeostasis (9). Particularly in the bone microenvironment, which is physiologically hypoxic, mechanisms regulating cellular adaptation to oxygen-poor conditions are crucial (10). The main orchestrator of the responses to hypoxia is HIF, a heterodimer transcription factor comprising a constitutive $\beta$ subunit (HIF- $\beta$ ) and an oxygen-regulated $\alpha$ subunit (HIF- $1 \alpha$ or HIF$2 \alpha$ ). In oxygen-rich conditions, HIF prolyl hydroxylases (PHDs) hydroxylate specific residues in the HIF- $\alpha$ protein, rendering it a substrate for the E3 ubiquitin ligase von Hippel-Lindau (VHL) and a target for proteasomal degradation. When oxygen levels drop below a critical level, HIF- $\alpha$ is not hydroxylated and degraded, but instead accumulates in the cell, liaises with HIF- $\beta$, and induces a hypoxia-triggered transcriptional program.

The key HIF target genes help cells face the challenges of low tissue oxygen, with 2 major mechanisms standing out. First, HIFregulated genes such as VEGF and erythropoietin (EPO) increase the tissue oxygen supply by inducing angiogenesis and erythropoiesis (9). Genetic studies in mice documented that also in osteoblast lineage cells, HIF directly regulates VEGF expression, thereby inducing blood vessel growth and bone formation and rendering 
the hypoxia signaling pathway the first recognized coupler of angiogenesis and osteogenesis $(10,11)$. The HIF pathway has also been shown to directly regulate EPO expression in osteoblasts, modulating erythropoiesis in the local hematopoietic BM environment (12).

Second, hypoxia-regulated transcription activates genes and pathways that reduce oxygen consumption and the cellular dependence on oxygen, including by mediating a bioenergetic switch from oxidative phosphorylation to glycolysis as major route of ATP production (9). This shift involves direct HIF-mediated upregulation of glycolytic enzymes such as pyruvate dehydrogenase kinase 1 (PDK1) and lactate dehydrogenase A (LDHA), promoting the conversion of pyruvate into lactate, and of glucose transporters (GLUTs, particularly GLUT1) to enhance glucose uptake, thereby compensating for the energy inefficiency of glycolysis. A similar metabolic reprogramming is also prominent in cancer cells, which commonly metabolize glucose by glycolysis even in the presence of oxygen, a phenomenon known as the Warburg effect or aerobic glycolysis $(13,14)$. Correspondingly, HIF-induced regulation of cellular glucose metabolism is being intensely investigated, with promising therapeutic potential in cancer and metastasis (15).

At present, relatively little is known about the metabolic pathways and substrates preferentially used by osteoblast lineage cells. Moreover, these may change throughout their lifespan and differentiation progress, and are likely context dependent $(16,17)$. Recent in vivo work using genetically modified mouse models has been actively investigating this topic, and revealed a prominent role for glucose metabolism during osteoblast differentiation (18, 19). Moreover, aerobic glycolysis has been implicated as an important driver of bone formation and shown to be regulated by osteoanabolic pathways involving Wnt-LRP5 signaling, parathyroid hormone, and HIF (20-23).

In this study, we show that the hypoxia signaling pathway directs cellular metabolism in osteoblast lineage cells, with HIFtarget gene expression potently stimulating glycolysis and glucose consumption. Intriguingly, our findings further indicate that the repercussions of this metabolic regulation in osteoblasts extend beyond the bone environment, to the control of whole-body energy metabolism. Specifically, we found that deletion of $V h l$ in osteoprogenitors, a model of persistent HIF activation, promoted glycolysis and glucose utilization by osteolineage cells. This led to an overall increase in glucose uptake from the circulation by the skeleton, which in turn correlated with reduced blood glucose levels in the mutant mice. The hypoglycemic phenotype was associated with an increased glucose tolerance that could not be explained through the known endocrine actions of osteocalcin and insulin, but which could be pharmacologically rescued by administration of a glycolysis inhibitor. This suggests that increased glucose usage by osteolineage cells can, possibly directly, stimulate systemic glucose clearance and improve glucose tolerance, and even lead to a sustained decrease in the blood glucose levels and to decreased peripheral fat accumulation. Strikingly, a parallel was seen with cancer patients carrying glucose-avid bone metastases, who also showed an inverse correlation between the local glucose uptake in the tumor lesions and global blood glucose levels, further underscoring the power of the Warburg effect.

Altogether, our findings suggest that hypoxia signalinginduced excessive glycolysis in osteolineage cells can lower systemic glucose levels by increasing glucose utilization by the skeleton. This simple yet unexpected concept brings a potential new angle to the sophisticated integration of the skeleton in global nutrient homeostasis, and may have broad clinical impact with regard to bone and metabolic disorders, as well as in cancer pathology and therapy.

\section{Results}

Vhl ablation in osteoprogenitors causes increased bone density and cortical porosity, along with hypervascularization and alterations in the BM environment. To investigate the impact of HIF signaling in osteoprogenitors and the osteoblast lineage cells derived thereof, Osx(SP7)-Cre:GFP mice (24) were crossed with $V h l$ floxed mice (25). Osx-GFP:Cre ${ }^{T G /+} \mathrm{Vhl}^{+/+}$mice were used as the control group. Genetic targeting of $V h l$ precludes the oxygen-dependent inactivation of HIF, thus representing a model of constitutive HIF activity and hypoxia signaling pathway responses, as documented previously $(11,12,26)$.

Vhl conditional knockout (cKO) mice displayed reduced skeletal growth (Figure 1A and Supplemental Figure 1A; supplemental material available online with this article; https://doi.org/10.1172/ JCI97794DS1) and a marked high bone mass phenotype at postnatal stages, characterized by a progressive accumulation of trabecular bone even at advanced age, as shown by micro-CT of the long bones (Figure 1, B-D). Excessive trabeculae extended far into the diaphyseal bone shaft, associated with thinner, highly porous, trabecularized cortical bone (Figure 1, B-F). High bone volume was also evident in the vertebrae (Figure 1G). Overall, bones in the mutant mice were heavier relative to BW than in controls (Supplemental Figure 1B). In line with previous reports on comparable mouse models $(11,12)$, Vhl cKO bones showed elevated expression of the direct HIF target genes Vegf and Epo (Figure 1H), associated with skeletal hypervascularization, BM fibrosis, and splenomegaly (Figure 1I and Supplemental Figure 1C).

Inactivation of Vhl in osteoprogenitors leads to an expanded pool of early osteolineage cells and a low bone remodeling status in adult mice. We further documented the bone remodeling status of the mice by analyzing bone formation and osteoblast differentiation on the one hand and bone resorption by osteoclasts on the other. Static, dynamic, and cellular histomorphometry indicated that by 12 weeks of age, the high bone mass phenotype was associated with substantially decreased active bone formation and mineralization activity as determined by calcein labeling (Supplemental Figure 2 and Figure 2, A and B), along with reduced presence of fully differentiated cuboidal osteoblasts on the bone surfaces of $V h l \mathrm{cKO}$ mice compared with controls (Figure 2C). In line therewith, differentiation and mineralization of $\mathrm{Vhl}$-deficient osteoblasts were reduced in vitro (Supplemental Figure 3). Expansion of the pool of early osteolineage cells in vivo was further supported by the increased presence of Osx-expressing cells (Figure 2D), increased expression of the early osteogenic cell marker runt-related transcription factor 2 (Runx2), and strongly decreased expression of the late osteoblast and osteocyte markers osteocalcin (Ocn) and sclerostin (Sost) (Figure 2E) in bones of $\mathrm{Vhl}$ cKO mice. Overall, abundant yet quite disorganized collagen and osteoid deposition was observed in the mutant bones by sirius red and van Gieson staining (Figure 2, F and $\mathrm{G})$. Impaired maturation and turnover of the bone matrix were also 

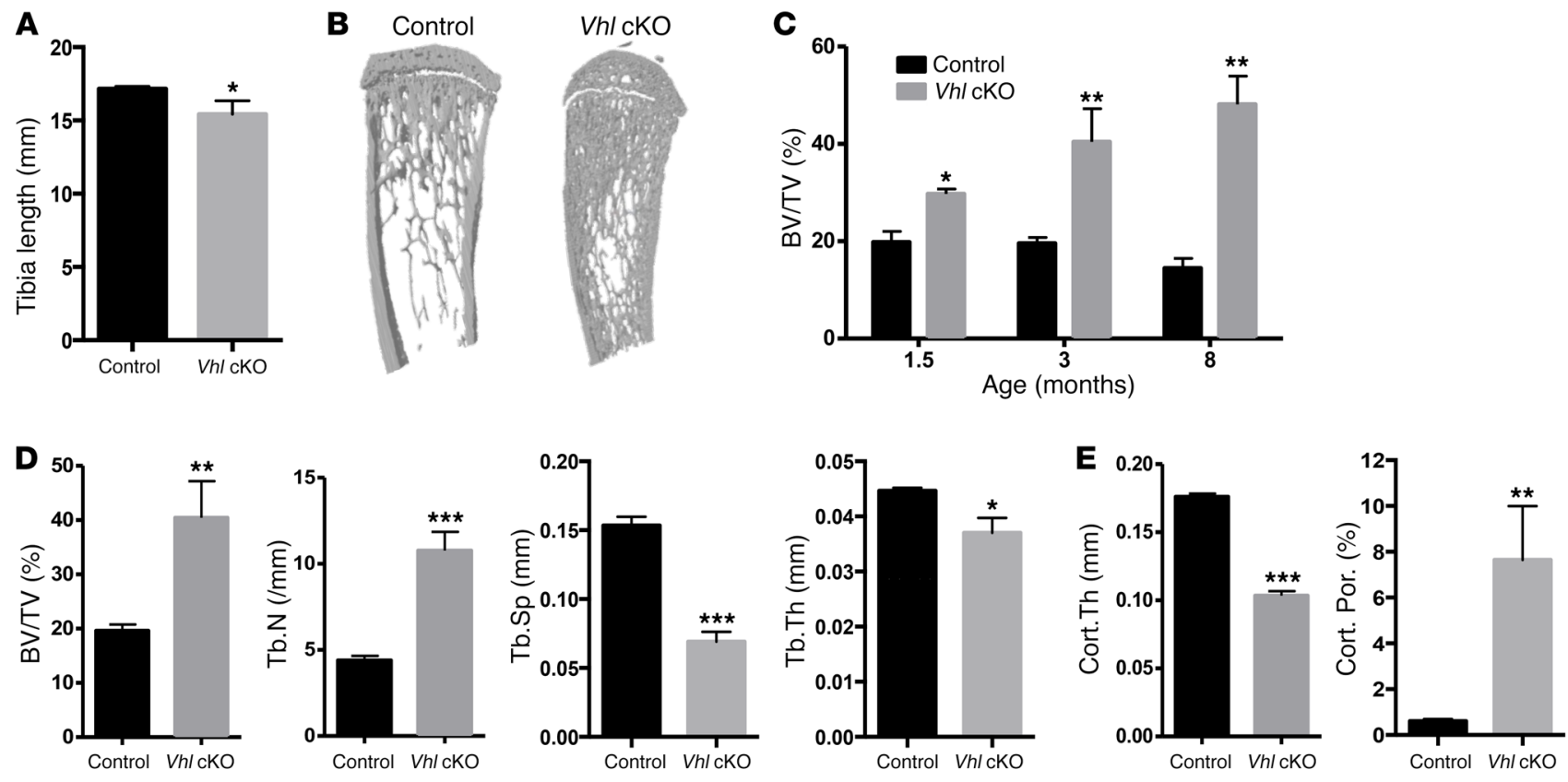

$\mathbf{F}$
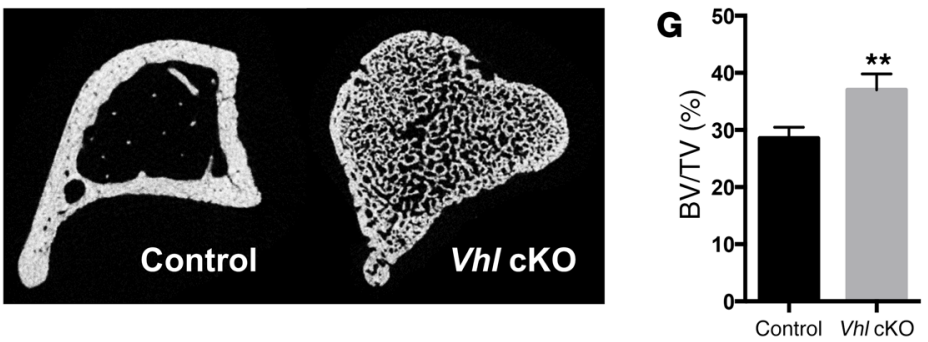
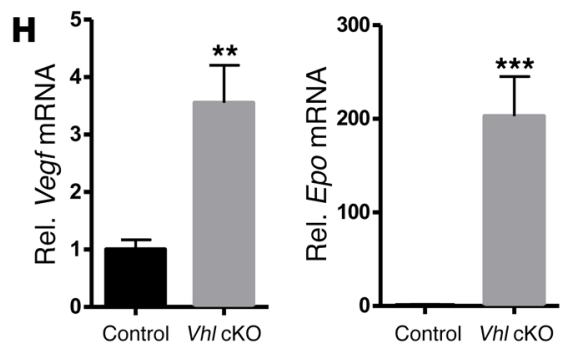

I
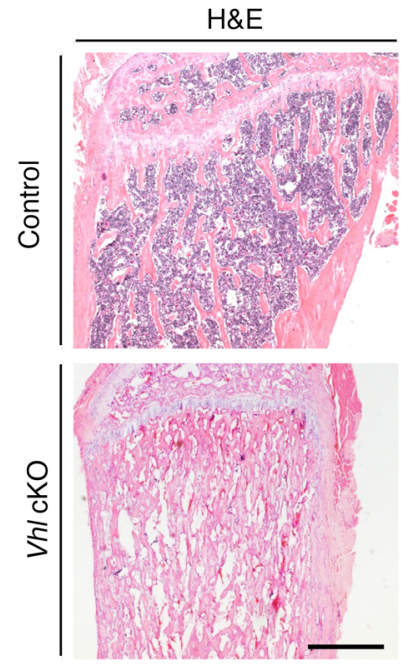

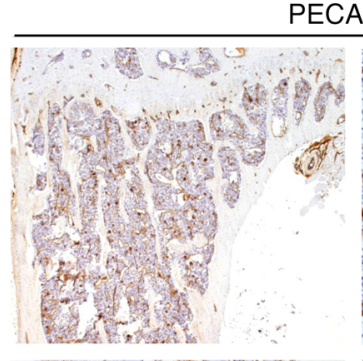

PECAM-1
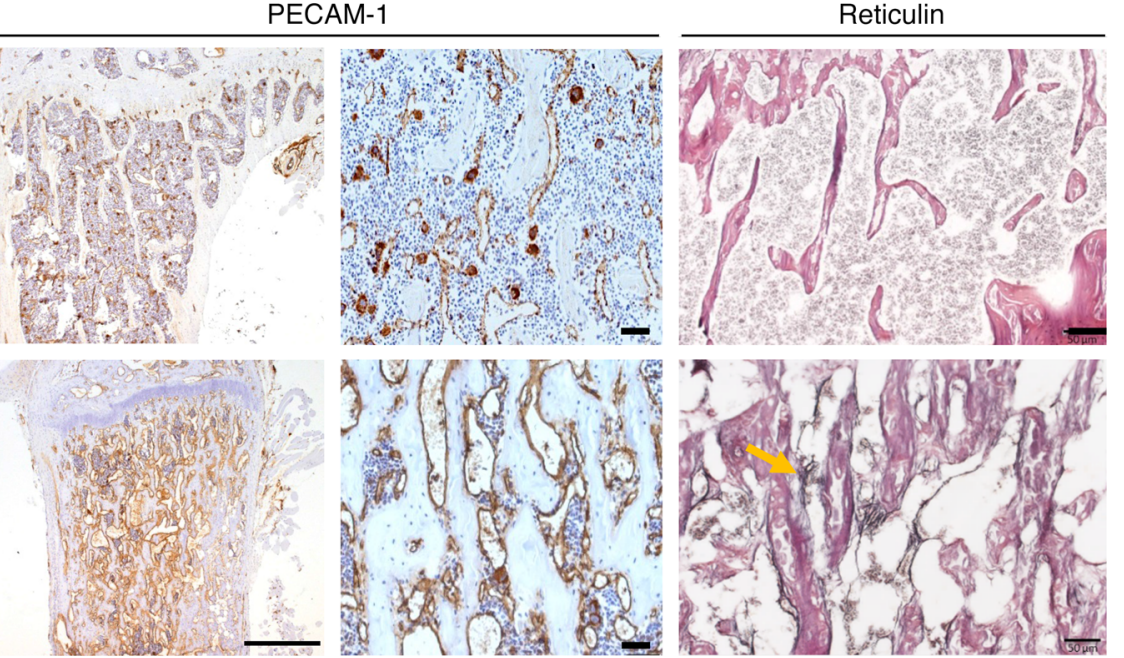

Figure 1. High bone mass and skeletal abnormalities in mice lacking $\mathbf{V h l}$ in osteoblast lineage cells. (A) Tibia length at 12 weeks ( $n=4-7$ per genotype). (B) Representative 3D micro-CT reconstructions of tibias from 12-week-old mice. (C) Bone volume relative to tissue volume (BV/TV, in \%) determined by micro-CT at the indicated ages ( $n=3-5$ /group). ( $\mathbf{D}$ and $\mathbf{E}$ ) Micro-CT analysis of the trabecular (D) and cortical (E) tibia regions at 12 weeks $(n=3-5)$, showing BV/TV; trabecular number (Tb.N), separation (Tb.Sp), and thickness (Tb.Th); and cortical thickness (Cort.Th) and porosity (Cort.Por). (F) Representative transverse micro-CT section of the tibia. (G) Vertebral BV/TV determined by micro-CT $(n=4)$. (H) Relative (Rel.) mRNA levels of Vegf and Epo in full bones of 12-week-old mice $(n=7)$. (I) Tibia histology showing H\&E staining, PECAM-1 IHC for blood vessels (including magnifications), and reticulin-positive fibers (black stain and yellow arrow) indicative of BM fibrosis in Vhl cKO bones. Scale bars: $500 \mu \mathrm{m}$ (H\&E; PECAM-1 left), $50 \mu \mathrm{m}$ (PECAM-1 right; reticulin). Graphs represent mean $\pm \mathrm{SEM}$, and ${ }^{*} P<0.05,{ }^{* *} P<0.01,{ }^{* *} P<0.001$ by Student's $t$ test between genotypes. 
A

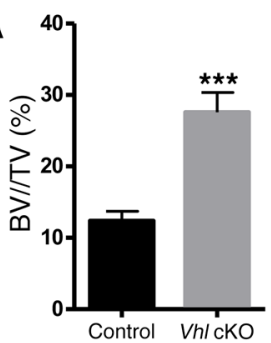

D

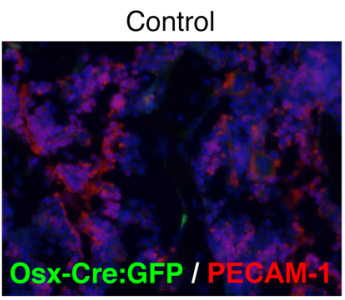

$\mathbf{F}$

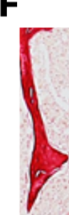

Sirius Red
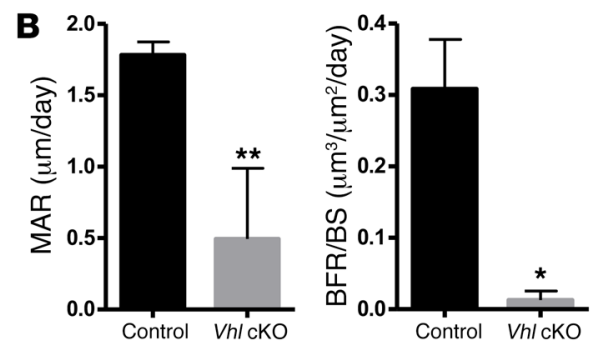
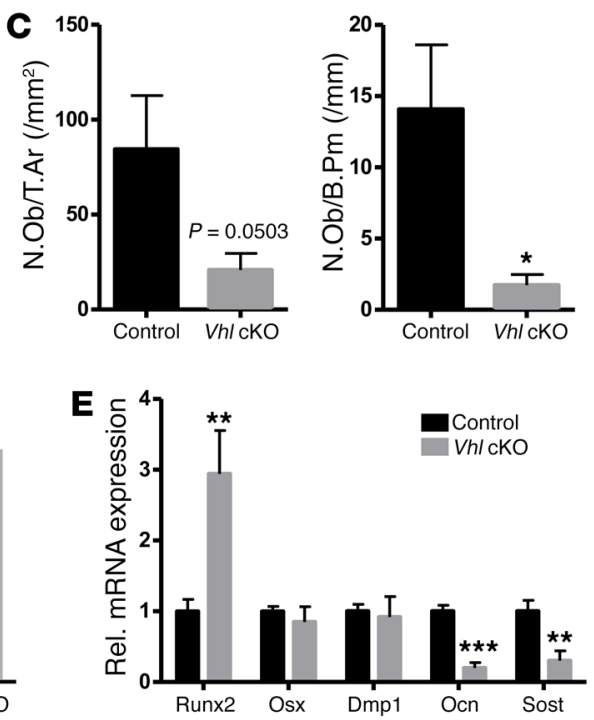

G $\mathrm{Vhl} \mathrm{cKO}$
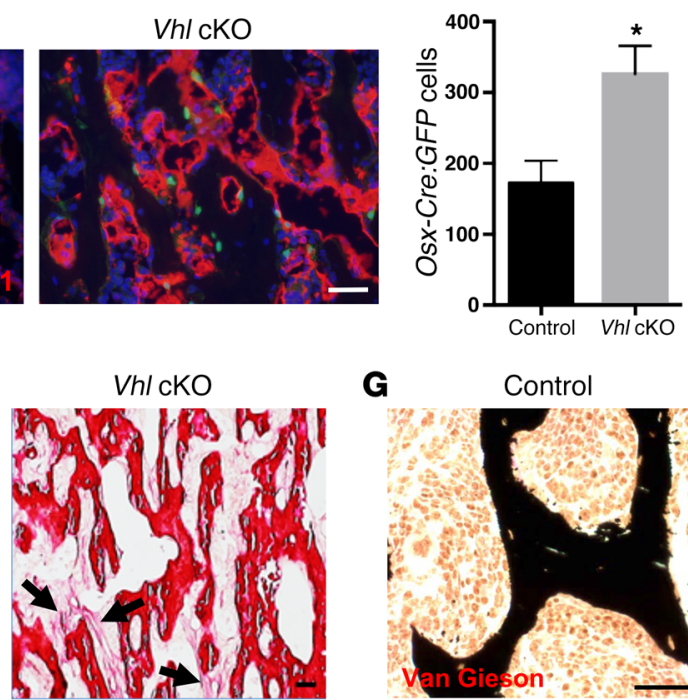
Control

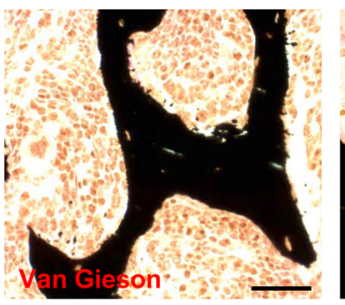
$\mathrm{Vhl}$ CKO

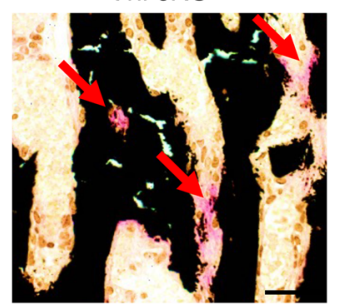

H

Control

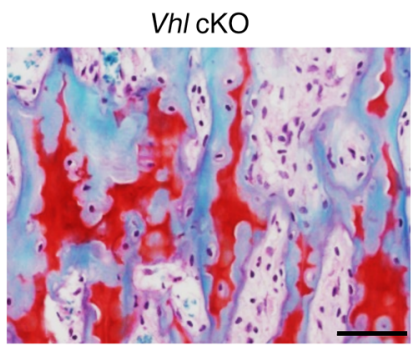

I
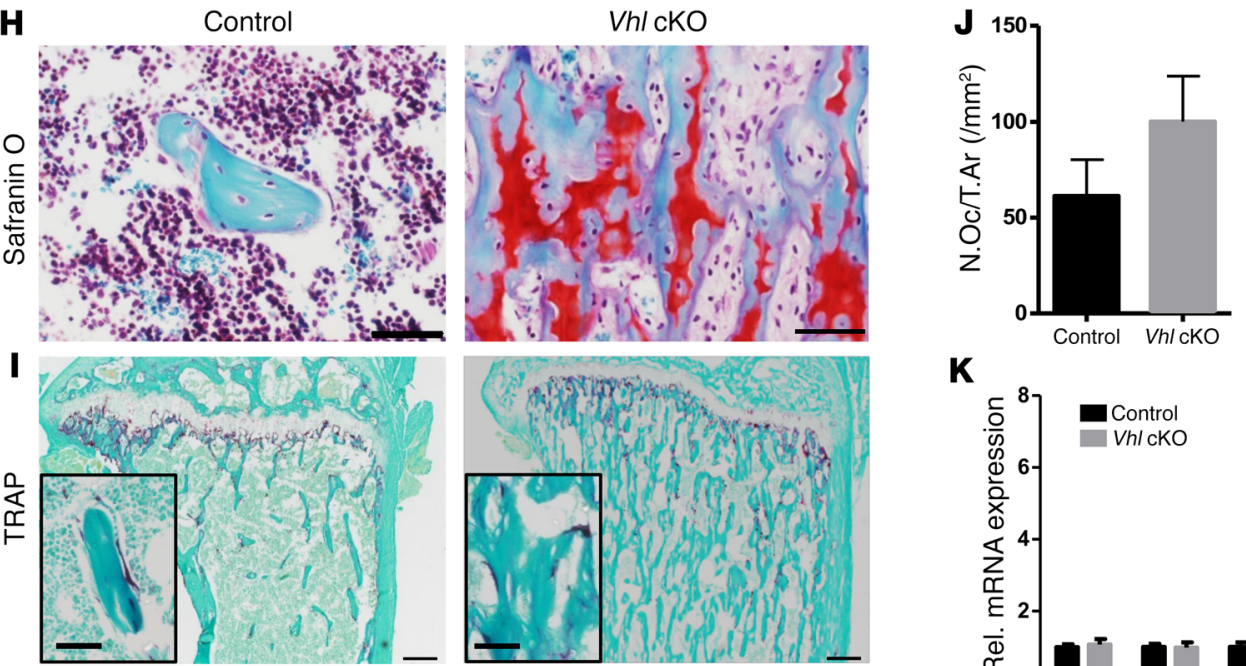

K
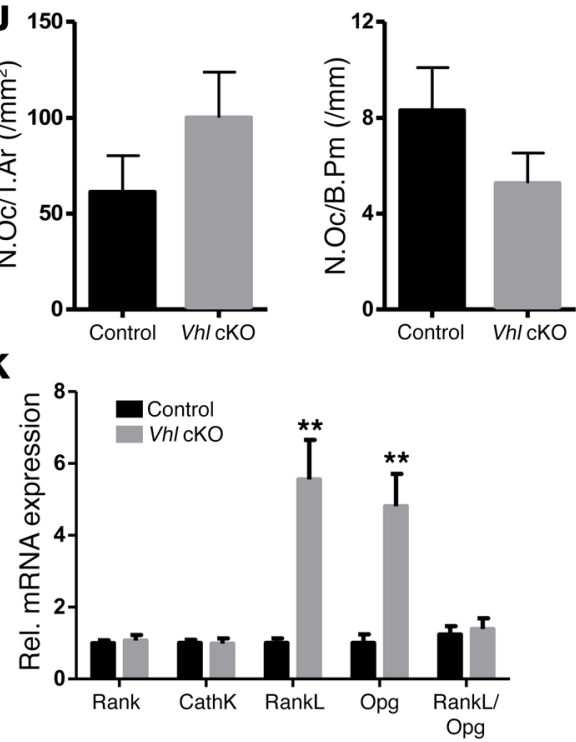

$\mathbf{L}$
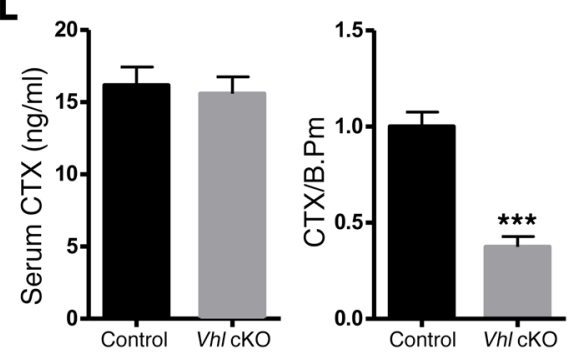

M

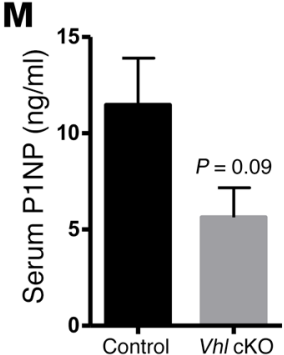

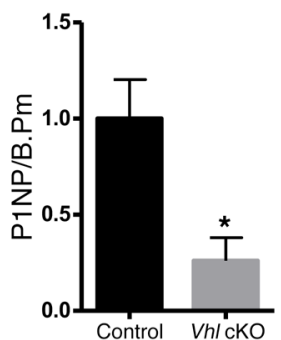


Figure 2. Inactivation of $\mathrm{Vhl}$ in osteoprogenitors leads to an expanded pool of early osteolineage cells and a low bone turnover status in adult mice. (A) BV/TV (\%) as quantified on von Kossa-stained sections $(n=8)$. (B) Mineral apposition rate (MAR) and bone formation rate corrected for bone surface (BFR/BS) quantified in mice injected with calcein twice with a 3-day interval $(n=3-7)$. (C) Numbers of mature, cuboidal osteoblasts located on the bone trabecular surface, expressed as absolute numbers (N.Ob in the region of interest, per unit of tissue area [T.Ar], left) or relative to bone perimeter (N.Ob/B.Pm, right) $(n=7-8)$. (D) Co-IHC for PECAM-1 and GFP (left) and number of Osx-Cre:GFP+ cells (right) $(n=5)$. Scale bar: $50 \mu \mathrm{m}$. (E) mRNA levels of the osteoblastogenesis markers Runx2, Osx, dentin matrix acidic phosphoprotein 1 (Dmp1), Ocn, and Sost $(n=7-8)$. (F) Representative sirius red-stained sections showing collagen fibers dispersed in the inter-trabecular BM environment (arrows) in mutant mice $(n=3)$. Scale bars: $100 \mu \mathrm{m}$. (C) von Kossa/van Gieson staining ( $n=$ 4), revealing mineralized bone (black) and nonmineralized osteoid (red, arrows). Scale bars: $50 \mu \mathrm{m}$. (H) Safranin 0 staining $(n=5)$. Scale bars: 50 $\mu \mathrm{m}$. (I) Tartrate-resistant acid phosphatase (TRAP) staining for osteoclasts (red, see magnification in inset) $(n=6-8)$. Scale bars: $200 \mu \mathrm{m}$. (J) Number of TRAP+ osteoclasts (N.Oc) in absolute terms per T.Ar (left) and relative to B.Pm (right) ( $n=6-8)$. (K) mRNA expression of osteoclast-specific markers (Rank, CathK) and osteoclastogenesis regulators (RankL, Opg) $(n=7)$. (L) Serum CTX values, expressed in absolute terms (left, $n=13-18$ ) or corrected for B.Pm (right, $n=5-7$ ). (M) Serum P1NP values, in absolute terms (left, $n=11-18$ ) and corrected for B.Pm (right, $n=3-6$ ). Graphs represent mean $\pm \mathrm{SEM}$, and ${ }^{*} P<0.05,{ }^{* *} P<0.01,{ }^{* *} P<0.001$ by Student's $t$ test between genotypes.

indicated by safranin $\mathrm{O}$ staining, which revealed abundant remnants of cartilage matrix within the trabecular bone structures of Vhl cKO mice, extending abnormally deep into the diaphysis (Figure $2 \mathrm{H}$ ). While sustained cartilage remnants are generally indicative of reduced resorptive activity, we could not detect significant alterations in the number of osteoclasts (Figure 2, I and J) or in the expression levels of osteoclast-specific marker genes such as Rank and cathepsin K (CathK), in Vhl cKO bones (Figure 2K). The expression levels of Rankl and osteoprotegerin (Opg), prime regulators of osteoclastogenesis expressed by osteoblast lineage cells, were both significantly increased in $\mathrm{Vhl}$ cKO bones compared with controls, yet the overall $\mathrm{Rankl} / \mathrm{Opg}$ ratio remained indifferent (Figure $2 \mathrm{~K}$ ). Last, the serum procollagen type $1 \mathrm{~N}$-terminal propeptide (P1NP) and C-terminal telopeptide (CTX) levels, respective markers of bone formation and resorption, were not significantly altered in $\mathrm{Vhl} \mathrm{cKO}$ versus control mice at 12 weeks of age; however, when corrected for trabecular bone perimeter, both parameters were significantly decreased in the mutant mice (Figure 2, L and M), in line with the overall presumption of a relatively low bone turnover status in adult $V h l \mathrm{cKO}$ mice.

Vhl cKO mice are lean, despite reduced physical activity, and display hypoglycemia and increased glucose tolerance. Surprisingly, constitutive deletion of VHL in osteoprogenitors was associated with distinct alterations in whole-body homeostatic processes. $V h l$ cKO mice showed markedly low BWs compared with littermate control mice from 3 weeks of age onward, and they appeared to be resistant to age-related weight gain, with the deviation in BW between the genotypes increasing throughout postnatal life (Figure 3A). The mutant mice had a lean appearance, displaying greatly reduced abdominal and subcutaneous fat (Figure 3, B and C), associated with reduced circulating levels of leptin but without changes in circulating adiponectin or in serum triglycerides
(Supplemental Figure 4, A-C). Indirect calorimetry showed normal food intake, oxygen consumption, and heat production in $\mathrm{Vhl}$ cKO mice, in the face of a significant decrease in ambulatory activity, suggesting that the basal metabolic rate was increased in the mutant mice (Figure 3, D and E). Respiratory exchange ratio (RER) values were around 0.8 in both genotypes (Figure 3F). Serum analysis indicated consistently lower blood glucose levels in $V h l c K O$ mice, both in random-fed and fasted conditions, from 6 weeks of age onward (Figure 3, G and H, and Supplemental Figure 4D). The hypoglycemic phenotype was accompanied by enhanced clearance of glucose from the blood following i.p. glucose injection during a glucose tolerance test (GTT), and an overall increase in glucose tolerance at the age of 6 weeks (Supplemental Figure $4 \mathrm{E}$ ) and 12 weeks (Figure 3I). Likely as a secondary consequence of the persistently lower glycemia, the rate-limiting gluconeogenesis enzyme phosphoenolpyruvate carboxykinase (PEPCK) was upregulated in the liver and muscle of constitutive $V h l$ cKO mice (Figure 3J), suggesting compensatory gluconeogenesis, and glycogen stores were virtually abolished in their livers (Figure $3 \mathrm{~K}$ ).

Unexpectedly, in light of the reduced glycemia and increased glucose tolerance, no alterations were observed in the serum insulin levels of $V h l$ cKO mice compared with controls in random-fed or fasted conditions (Figure 3L). Also, the insulin-producing $\beta$ cell area in the pancreas (Supplemental Figure 5) and endogenous insulin secretion in response to glucose injection (glucose-stimulated insulin secretion test [GSIS]) (Figure 3M) were normal. Moreover, insulin tolerance tests (ITTs) demonstrated a reduction in insulin sensitivity in $V h l$ cKO mice (Figure $3 \mathrm{~N}$ ). Yet, even though $V h l \mathrm{cKO}$ mice appeared to be less insulin sensitive than controls, calculation of the homeostatic model assessment of insulin resistance (HOMA-IR) did not classify them as insulin resistant (Figure 3O). Furthermore, no evidence was found for peripheral insulin resistance or altered insulin signaling in the liver or skeletal muscle of $\mathrm{Vhl}$ cKO mice, as indicated by gene expression analysis of insulin targets, enzymes involved in carbohydrate metabolism, and markers of muscle fiber composition (Supplemental Figures 6 and 7), and as corroborated by normal glucose uptake in these insulin target tissues (Supplemental Figure 6D and see below, Increased skeletal glucose uptake correlates with reduced glycemia levels).

Altogether, these data indicate that constitutive skeletaltargeted $V h l \mathrm{cKO}$ mice have a high bone mass, improved glucose metabolism, and a lean body, all in the face of normal food intake and reduced physical activity.

Mice with constitutive or induced skeletal Vhl deletion develop high bone mass, low glycemia levels, and enhanced systemic glucose tolerance, not accounted for by insulin or osteocalcin. As described above, constitutive $V h l$ cKO mice were in a state of persistent low glycemia with increased glucose tolerance from juvenile ages onward, which appeared to be associated with a failure to build up energy stores such as those normally provided by peripheral fat and liver glycogen. This observed lipodystrophy/lipoatrophy consequently contributed to the complexity of interpreting the metabolic phenotype in this constitutive model. Therefore, we additionally generated a postnatally induced $V h l \mathrm{cKO}(\mathrm{PN}-V h l \mathrm{cKO})$ mouse model, by preventing the recombination of the floxed $V h l$ gene during development and until postnatal week 3 through the administration of doxycycline, which silences the tetracycline-off 

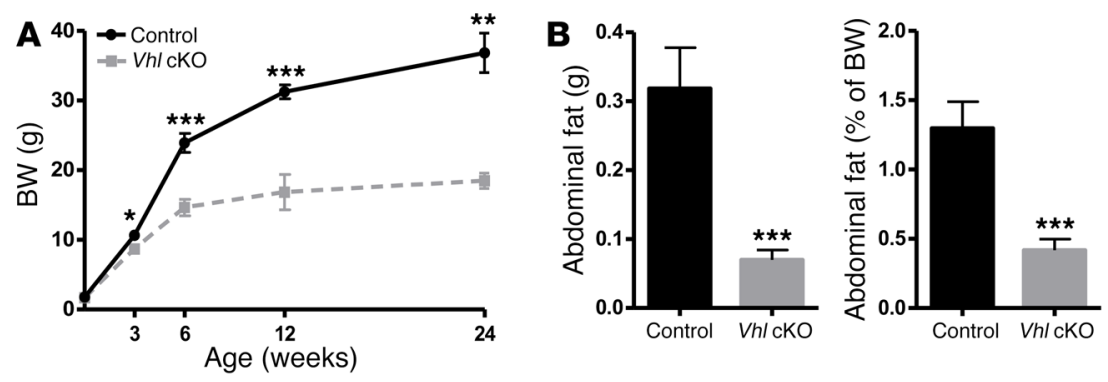

C
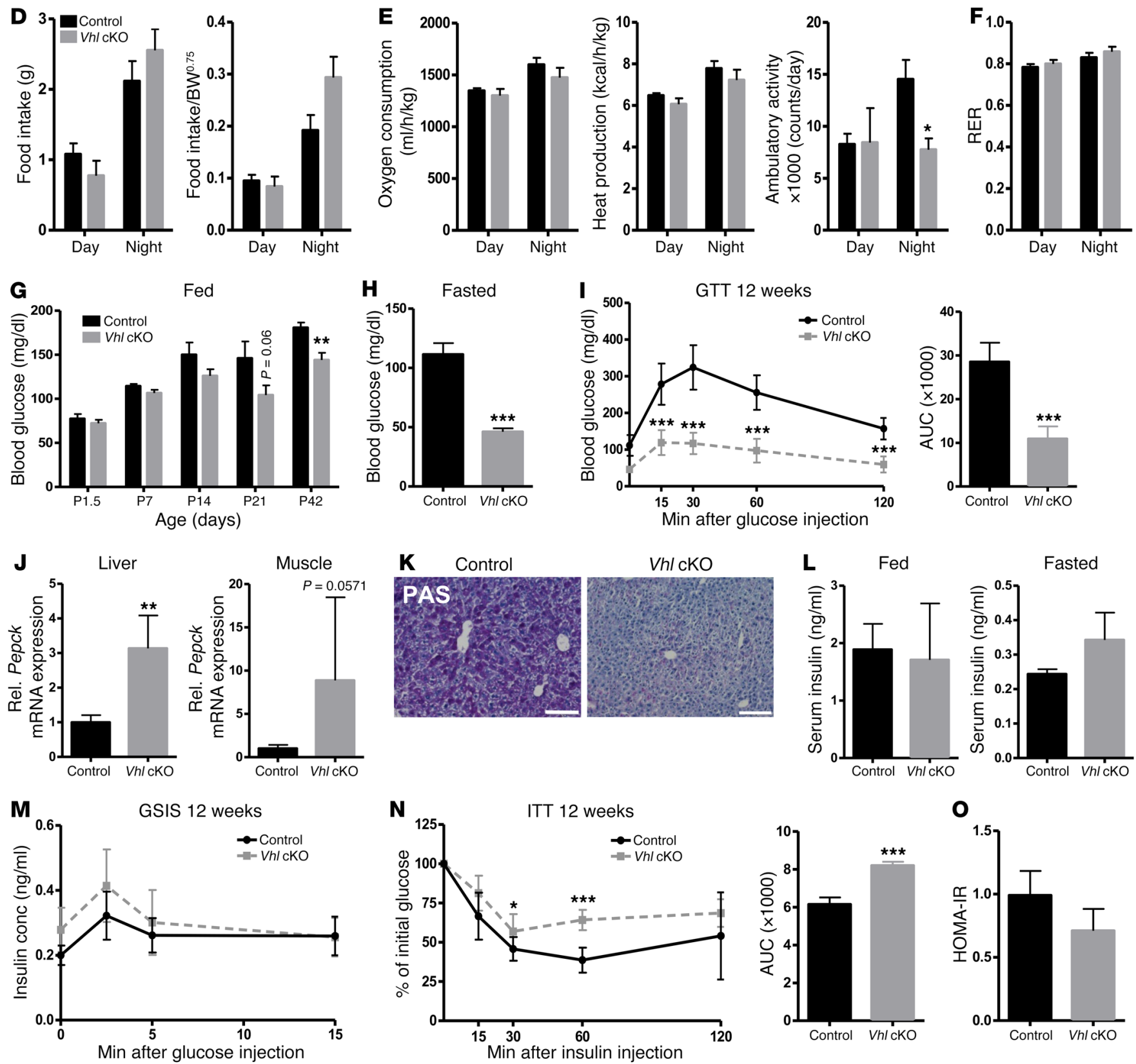
Figure 3. Osteoprogenitor-targeted VhI cKO mice are lean, despite normal food intake and reduced physical activity, and display a hypoglycemic phenotype with increased systemic glucose tolerance. (A) BW of control and constitutive $\mathrm{Vhl}$ cKO mice at the indicated ages ( $n=3-10 /$ group). (B) Abdominal fat mass as absolute values, in grams (left), and relative as percentage of BW (right) ( $n=10-14$ ) in 12-week-old male mice. (C) Representative $\mathrm{H} \& \mathrm{E}$-stained skin sections. Scale bar: $100 \mu \mathrm{m}$. Arrows point to subcutaneous fat. (D) Food intake over 2 days/nights, in absolute values (left) and corrected for the metabolic BW (BW ${ }^{0.75}$, BW raised to the three-quarter power, as commonly used to normalize energy metabolism data) (right) $(n=6-7)$. (E) Indirect calorimetry measurements of oxygen consumption, heat production, and ambulatory activity, corrected for $\mathrm{BW}^{0.75}(n=6-7)$. (F) RER $(n=6-7)$. (G) Blood glucose levels in random-fed state from P1.5 to P42 ( $n=6-8$ /group). (H) Blood glucose levels after overnight fasting at 12 weeks ( $n=7-9)$. (I) GTT and its quantification as AUC $(n=7-9)$. (J) Pepck mRNA levels in liver and muscle $(n=4)$. (K) PAS staining on liver, revealing glycogen content $(n=4)$. Scale bar: $100 \mu \mathrm{m}$. (L) Serum insulin levels in random-fed (left) and fasted (right) conditions $(n=4-9)$. (M) GSIS $(n=9)$. conc, concentration. (N) ITT and AUC quantification $(n=6-9)$. (O) HOMA-IR $(n=9)$. All analyses were performed on male control and constitutive $\mathrm{Vhl}$ cKO mice at 12 weeks of age, unless indicated otherwise. Graphs represent mean \pm SEM, and ${ }^{*} P<0.05,{ }^{* *} P<0.01,{ }^{* *} P<0.001$ by Student's $t$ test between genotypes, unless indicated otherwise.

system-containing (Tet-Off-containing) Osx-Cre:GFP transgene (24) (Figure 4A). In contrast to the constitutive $V h l$ cKO mouse, the induced mutant $\mathrm{PN}-V h l$ cKO mice appeared neither smaller nor leaner than control littermates, as evidenced by their normal BW and abdominal fat accumulation (Figure 4, B and C). Circulating levels of adiponectin were also unchanged in $\mathrm{PN}-\mathrm{Vhl} \mathrm{cKO}$ mice (Figure 4D), and their livers showed no alterations in mRNA expression levels of genes involved in glycolysis, gluconeogenesis (Pepck), and glycogen metabolism (Supplemental Figure 7). Interestingly though, from the age of 12 weeks and most evidently by 24 weeks, the PN-Vhl cKO mice recapitulated all other key features of the constitutive $V h l$ cKO model, as they displayed a marked high bone mass (Figure 4, E-H), reduced fasted blood glucose levels (Figure 4I), and increased glucose tolerance (Figure 4J) compared with control littermates, without alterations in serum insulin levels (Figure $4 \mathrm{~K}$ ) or insulin sensitivity (Figure $4 \mathrm{~L}$ ). This phenotype was documented in both male and female groups of mice (Figure 4 and Supplemental Figure 8). As in the constitutive model, the local alterations in the bones of $\mathrm{PN}-\mathrm{Vhl} \mathrm{cKO}$ mice were associated with increased expression of Vegf and Epo, skeletal hypervascularization, and splenomegaly (Supplemental Figure 9). Indirect calorimetry showed normal oxygen consumption, heat production, and ambulatory activity, but a significant increase in RER in the PN-Vhl cKO mutants, suggesting a slightly increased glucose metabolism (Supplemental Figure 10).

These data show that the local and systemic repercussions of constitutive $V h l$ deletion in osteolineage cells were recapitulated in a context of normal development and baseline physiology using a postnatal inducible genetic system. The $\mathrm{PN}-V h l \mathrm{cKO}$ mouse thereby provided an additional and simplified model to explore the role of the skeleton in the regulation of global glucose homeostasis.

Since these findings underscored a novel potential link between hypoxia pathway signaling in osteolineage cells and whole-body energy metabolism, we thoroughly checked the skeletal specificity of the genetic targeting strategy. The history of the Osx-Cre:GFP-mediated recombination was evaluated in sections of bone and soft tissues using the IRG transgenic reporter mouse, in which cells switch from red fluorescent protein to GFP expression following Cre-mediated recombination. As expected, the reporter readout in bone revealed Osx-Cre-targeted $\mathrm{GFP}^{+}$ cells in the hypertrophic chondrocyte regions of the growth plate, throughout the metaphysis and in the cortical and trabecular bone areas (Supplemental Figure 11A). The GFP signal corresponded with osteolineage cells on the bone surfaces as well as some $\mathrm{GFP}^{+}$ reticular cells dispersed within the BM stroma (Supplemental Figure $11 \mathrm{~A}$, middle) and the majority of bone-embedded osteocytes (Supplemental Figure 11A, bottom). Abundant Osx-Cre-targeted cells and progeny were observed in bones from $\mathrm{Vhl}$ cKO mice (Supplemental Figure 11B). When evaluating the IRG reporter readout of Osx-Cre activity in other tissues, including the spleen, liver, and intestine, we did not observe any $\mathrm{GFP}^{+}$cells (Supplemental Figure 11C). Furthermore, we quantified the mRNA levels of Vhl and a panel of highly responsive HIF target genes (phosphoglycerate kinase 1 [Pgk1], Epo, Glut1, and Vegf) in brain, liver, pancreas, and small intestine of control and constitutive $V h l$ cKO mice. No detectable upregulation was seen for any of these genes in the soft tissues derived from the mutant mice (Supplemental Figure 12A), whereas they all showed very strong upregulation in $V h l$-deficient osteoblasts and in bones derived from $V h l$ cKO mice (Supplemental Figure 12, B and C). The sensitivity of this approach and significance of this finding is underscored by the fact that these whole-bone samples contain only around $2 \%-2.5 \%$ osteolineage cells, with the majority of the sample representing the nontargeted hematopoietic cells of the BM (data not shown). When the OsxCre: $G F P^{+}$cells were selectively sorted from calvaria or long bones of control and $V h l \mathrm{cKO}$ mice by FACS, they revealed effective inactivation of the $V h l$ gene. As shown in Supplemental Figure 12D, $V h l$ mRNA levels were reduced (by up to $80 \%$ ) specifically in the OsxCre: $G F P^{+}$cell fractions derived from mutant mice and not in the Os $x$-Cre:GFP- cell fractions. Altogether these data indicate that $V h l$ recombination was efficient in the osteolineage cells of bone, but absent or marginal in the nonskeletal tissues tested.

Given that osteoblast-derived osteocalcin is the principal known mediator of the interplay between bone and energy metabolism, we next quantified the osteocalcin protein levels in the serum of mice with constitutive or induced conditional $V h l$ deletion. Interestingly, circulating osteocalcin levels were strongly reduced (up to $80 \%$ ) in $\mathrm{Vhl}$ mutant mice compared with controls (Figure 5, A and B), corresponding to the low bone remodeling status of the mice (see Figure 2). These low serum osteocalcin levels were in line with the substantial downregulation of Ocn mRNA expression in bones of $V h l \mathrm{cKO}$ and $\mathrm{PN}-V h l \mathrm{cKO}$ mice (Figure 5, $\mathrm{C}$ and D) and with reduced Ocn mRNA expression in Vhl-deficient primary osteoblasts (Figure 5E). However, these results were unpredicted in light of the hypoglycemic phenotype, since serum osteocalcin levels generally inversely relate to plasma glucose (3).

Altogether, our data indicate that skeletal-targeted $V h l \mathrm{cKO}$ mice, both the constitutive and the postnatally induced models, showed a high-bone-mass phenotype locally and low glycemia with increased glucose tolerance systemically. Intriguingly, the low glycemia and increased glucose tolerance in $\mathrm{Vhl}$ cKO mice could not be simply explained through increased insulin or osteo- 
A

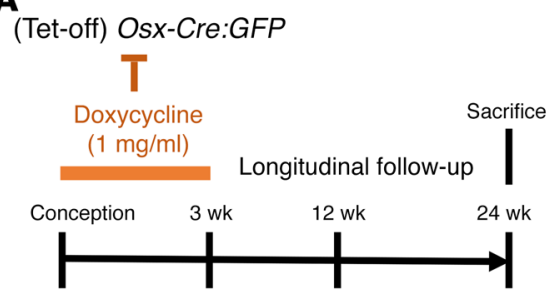

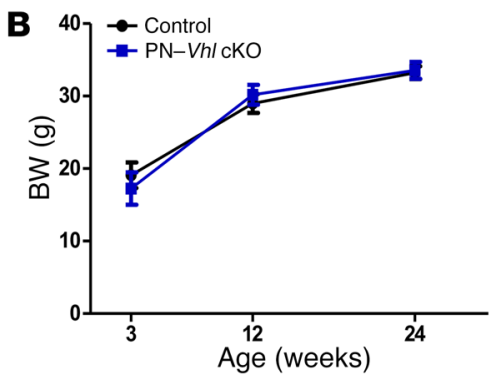
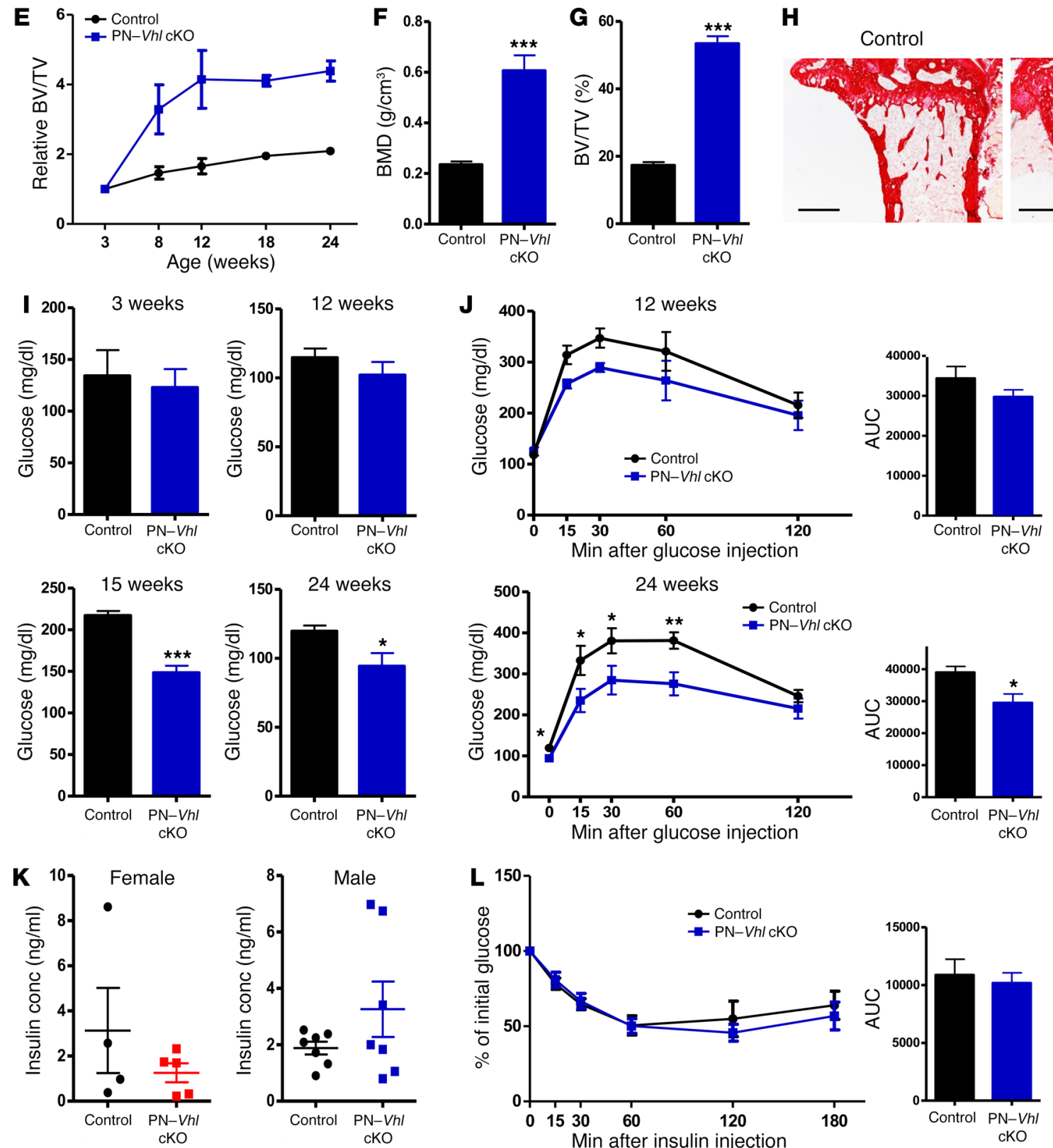
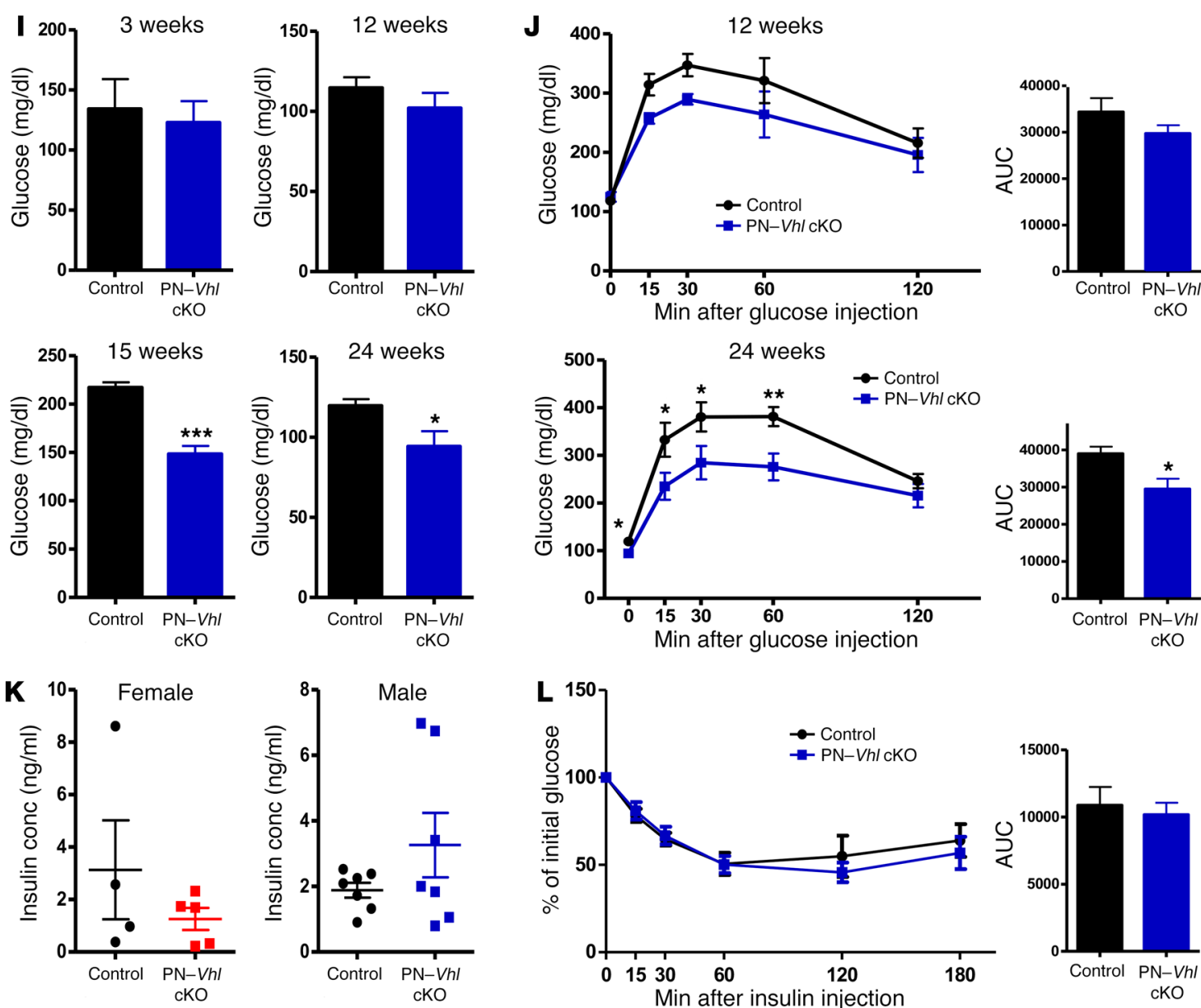

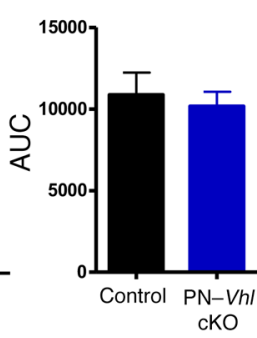

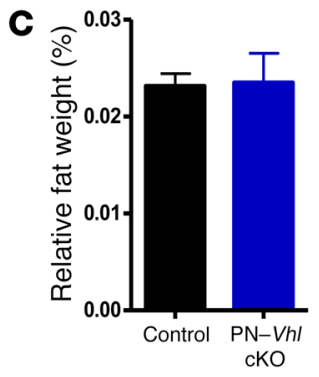

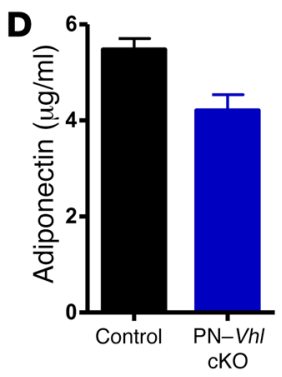

$\mathbf{H}$

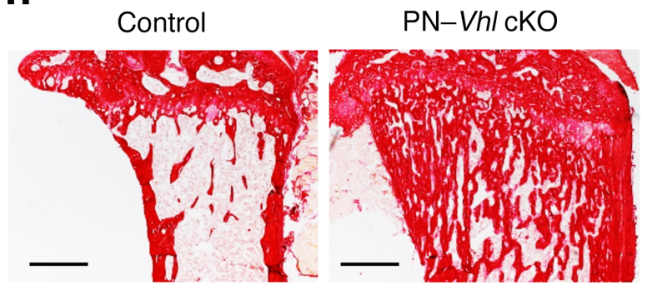

Figure 4. PN-Vhl cKO mice recapitulate the key skeletal and systemic features of the constitutive VhI cKO model while showing normal BW and fat mass. (A) Scheme of doxycyclin administration to silence Osx-Cre:GFP activity from conception until weaning. (B) BW of control and PN-VhI cKO mice at the indicated ages $(n=6-9)$. (C) Abdominal fat weight as percentage of BW at 24 weeks $(n=6-7)$. (D) Serum adiponectin levels at 24 weeks ( $n=7-8)$. (E) Relative BV/TV change over time analyzed by in vivo micro-CT. (F and G) Bone mineral density (BMD) (F) and BV/TV (\%) (G) determined by ex vivo micro-CT at 24 weeks $(n=7-8)$. (H) Representative sirius red-stained tibia sections (scale bars: $500 \mu \mathrm{m})(n=3)$. (I) Blood glucose levels at 3 weeks of age (overnight fast, $n=6$ ), 12 weeks (overnight fast, $n=6-8$ ), 15 weeks (3-hour fast, $n=6-8$ ), and 24 weeks of age (overnight fast, $n=7-8$ ). (J) GTT at 12 weeks ( $n=3-7$ ) and 24 weeks $(n=7-8)$, with corresponding AUC calculations. (K) Serum insulin levels in random-fed conditions at 24 weeks in females (left, $n=4-5)$ and males (right, $n=7)$. (L) ITT and corresponding AUC $(n=4-5)$. Graphs represent mean \pm SEM, and ${ }^{*} P<0.05$, ${ }^{* *} P<0.01$, ${ }^{* *} P<0.001$ by Student's $t$ test between genotypes, unless indicated otherwise. All data in B-J and L were obtained in male control and induced PN-Vhl cKO mice. Corresponding data from female groups are shown in Supplemental Figure 8. 

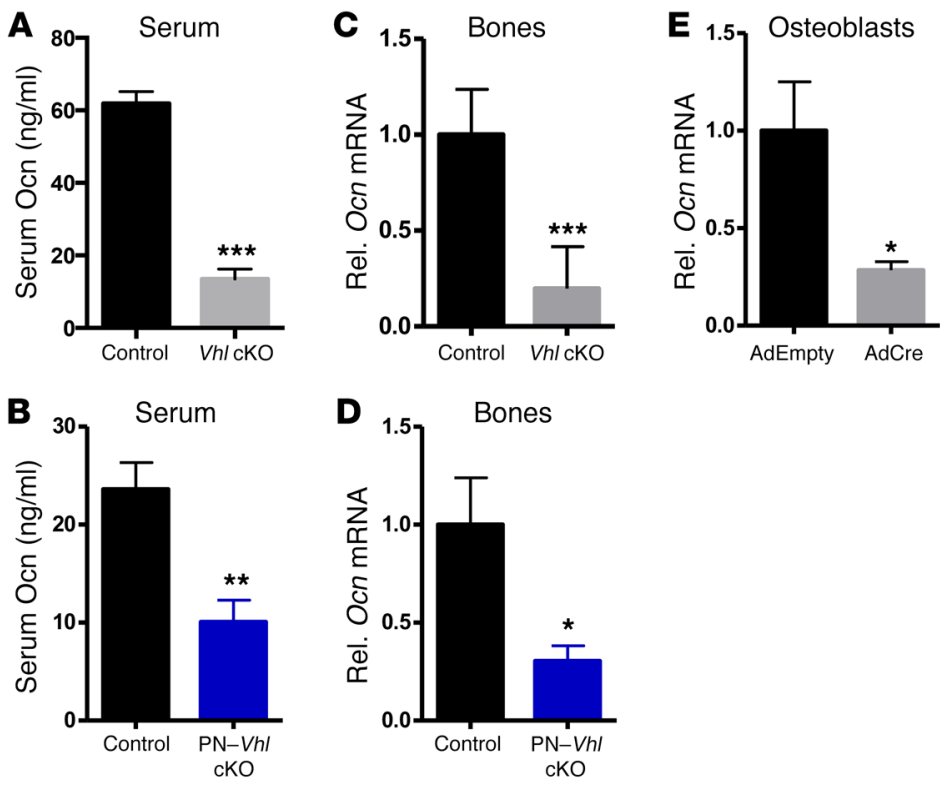

Figure 5. Vhl-deficient mice develop increased glucose tolerance and low glycemia despite greatly reduced production and levels of osteocalcin in their bones and serum. (A and B) Serum osteocalcin $(\mathrm{Ccn})$ levels in (A) control and constitutive $\mathrm{Vhl}$ cKO mice $(n=9-14)$ and (B) control and PN-Vhl cKO mice $(n=7-8$.). (C and D) Ocn mRNA levels in bones of (C) 12-week-old control and constitutive $\mathrm{Vhl}$ cKO mice $(n=7)$ and (D) 24-week-old control and PN-Vhl cKO mice $(n=$ 6-8). (E) Ocn mRNA levels in osteoblasts derived from $\mathrm{Vhl}$-floxed mice and transduced with AdEmpty or AdCre in vitro (also see Figure 6) $(n=5)$. All data were obtained in male mice. Graphs represent mean $\pm \mathrm{SEM}$, and ${ }^{*} P<0.05$, ${ }^{*} P<0.01,{ }^{* *} P<0.001$ by Student's $t$ test between genotypes, unless indicated otherwise. calcin signaling, indicating that an alternative mechanism must underlie the phenotype.

Excessive glucose uptake and glycolysis in Vhl-deficient osteoblasts. In search of the mechanism underlying the systemic phenotype of the $V h l$ cKO mice, we reverted to the primary target of our genetic strategy, the osteoblast, and used Vhllt/fl primary osteoblasts transduced in vitro with adenoviruses expressing Cre (AdCre) or carrying a control vector (AdEmpty/AdGFP) as a model system. The AdCre-treated cells showed over 90\% downregulated $V h l$ mRNA levels and effective HIF- $1 \alpha$ protein stabilization (Figure 6, A and B).

Consistent with the presence of consensus hypoxia-response elements (HREs, the recognition sites for HIF) in their regulatory sequences, the genes encoding the glycolysis-regulating enzymes PGK1, PDK1, and LDHA were significantly upregulated in $\mathrm{Vhl}$ deficient cells (Figure 6C). AdCre cells also displayed elevated expression of several GLUTs (Figure 6D), particularly GLUT1, the dominant glucose transporter in osteoblasts (18). In line with these changes at the gene expression level, Vhl-deficient cells showed increased uptake of glucose and production of lactate, as documented by analysis of conditioned culture media (Figure $6 \mathrm{E}$ ) and quantification of cellular uptake of 2-NBDG, a fluorescently labeled glucose analog (Figure 6F) that is not metabolized. Extracellular flux analysis confirmed that the mutant cells displayed a change in their bioenergetics, as evidenced by increased extracellular acidification rates (ECARs) (Figure 6G), reflecting the strongly increased lactic acid secretion, and decreased basal oxygen consumption rates (OCRs) (Figure 6H), indicative of reduced glucose oxidation. The response of AdCre-treated cells to mitochondrial stress test components (oligomycin, FCCP, and rotenone) indicated trends toward reduced oxygen consumption for ATP production $(P=0.065)$ and maximum respiration capacity (Figure 6I), yet no significant differences were seen in these circumstances. In line with these results, we documented slightly downregulated expression of mitochondrial biogenesis markers in Vhl-deficient cells (Figure 6J). Yet, despite the lowered mitochondrial respiration, the levels of ATP produced by AdCre-treated cells were normal (Figure 6K), as was the phosphorylation status of the cellular energy sensor AMPK (Figure 6L).

These data provide evidence that the uptake and energyinefficient glycolytic breakdown of glucose is greatly increased in $V h l$-deficient osteoblasts, to the extent that their reduced mitochondrial respiration is compensated for and cellular energy homeostasis is maintained.

Osteoblast lineage cells are prime glucose-consuming cells in the bone environment. The molecular alterations observed in vitro were confirmed in vivo: bones of $V h l \mathrm{cKO}$ and $\mathrm{PN}-\mathrm{Vhl} \mathrm{cKO}$ mice displayed increased mRNA expression of key glycolytic enzymes, most pronouncedly Pgk1 and Pdk1, and of Glut1 (Figure 7, A and B). Immunostaining confirmed an increased presence of GLUT1 in the mutant bones of both the constitutive and the postnatally induced models, particularly in osteoblast lineage cells on and around the bone surfaces (Figure 7, C and D). To visualize the uptake of glucose in situ, we next administered 2-NBDG to control and $V h l \mathrm{cKO}$ mice, and assessed the uptake and accumulation of the compound in the tibia and calvaria, harvested 5 minutes (data not shown) or 45 minutes (Figure 7E and Supplemental Figure 13, A and B) after the injection. These experiments revealed that osteoblasts lining the bone surfaces take up glucose quickly and abundantly, and represent by far the most glucose-avid cells in mouse bones, with relatively sparse uptake being detected in chondrocytes, hematopoietic cells, and osteocytes (Figure 7E, upper panels and Supplemental Figure 13A). The major glucoseconsuming cells included presumed immature osteogenic cells in the primary spongiosa (close to the growth plate) and in the trabecular bone microenvironment, flattened bone lining cells, and cuboidal mature osteoblasts on the trabeculae and on the cortical bone, which were overall particularly abundant in the mutant mice (Figure 7E, bottom panels).

These data suggest that osteoblast lineage cells, in particular osteoprogenitors and osteoblasts, are responsible for a substantial part of the skeletal glucose uptake. Alterations in their glucose utilization may consequently impact notably on the skeleton's overall glucose consumption. The increase in glycolytic pathway activation 

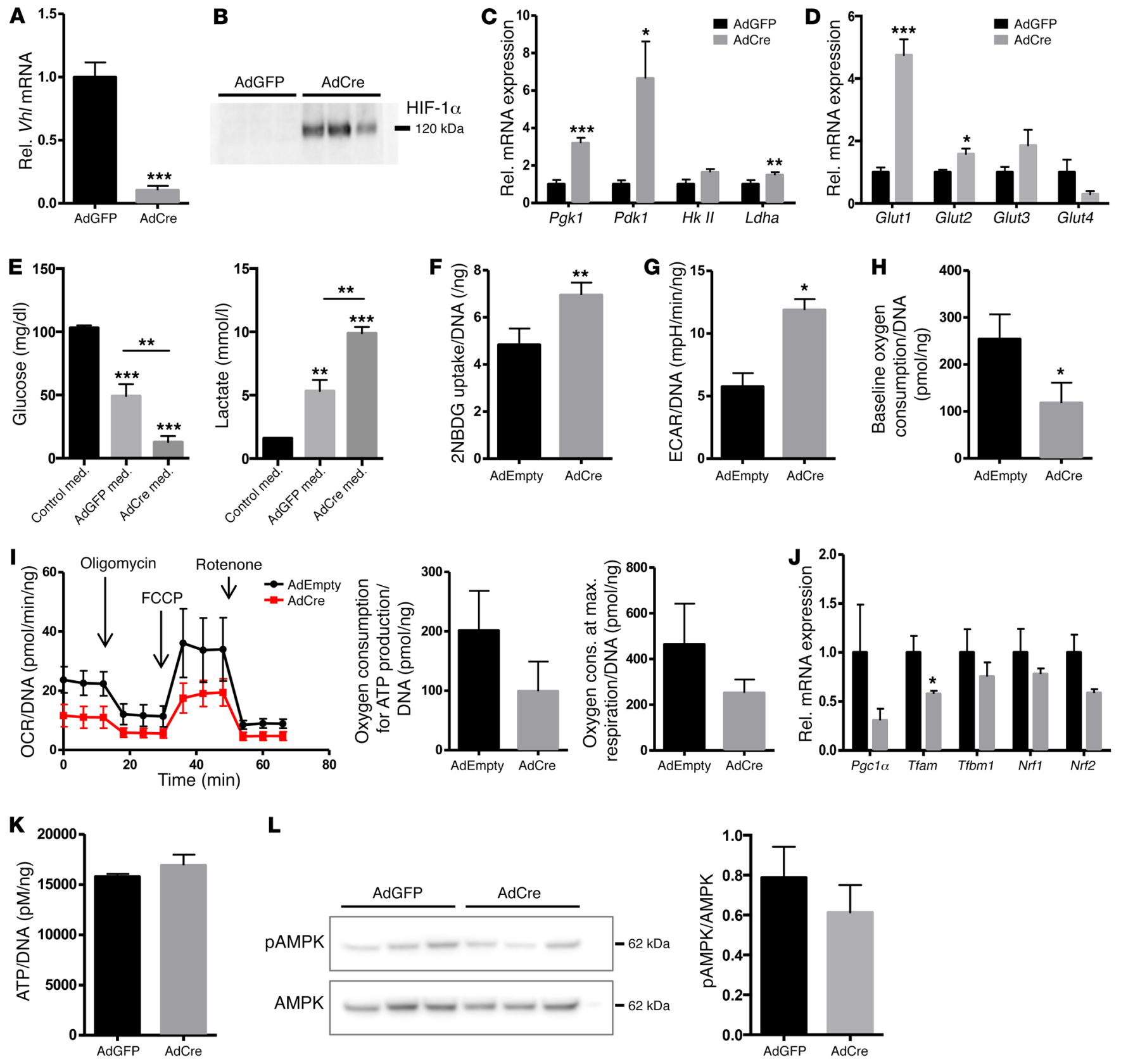

Figure 6. Vhl-deficient osteoblasts show increased glucose uptake and glycolysis. (A) Relative mRNA expression levels of Vhl in cultured primary osteoblasts derived from Vh/flff mice and transduced with adenoviruses expressing GFP (AdGFP) or Cre (AdCre) ( $n=5$ paired independent cell pools). (B) Western blot for HIF-1 $\alpha$ confirming its effective stabilization in Vhl-deficient cells (AdCre) compared with control cultures (AdGFP) ( $n=3$ ). (C and D) mRNA levels of (C) Pgk1, Pdk1, hexokinase 2 (HkII), and Ldha, and (D) Glut1-4 in AdGFP- versus AdCre-transduced Vh/flfl osteoblasts ( $n=5$ cell pools). (E) Glucose (left) and lactate (right) concentration in unconditioned (black bars) and 24-hour-conditioned medium $(n=5-6)$. med., medium. (F) 2-NBDG uptake in primary osteoblasts (normalized for DNA content of the well, $n=3$ ). (G-I) Extracellular flux analysis of primary osteoblasts $(n=5)$, showing $(\mathbf{G})$ ECAR, (H) basal oxygen consumption, and (I) OCR during a mitochondrial stress test including the oxygen consumption needed for ATP production and at maximum respiration, all normalized for DNA content. (J) Relative mRNA expression levels of mitochondrial biogenesis genes in control (AdGFP) versus Vhl-deficient (AdCre) cultured osteoblasts $(n=5)$, showing peroxisome proliferator-activated receptor $\gamma$ coactivator $1 \alpha(P g c 1 \alpha)$, transcription factor A, mitochondrial (Tfam), transcription factor B1, mitochondrial (Tfbm1), nuclear respiratory factor 1 (Nrf1) and 2 (Nrf2). (K) ATP production, normalized for DNA ( $n=4)$. (L) Western blot for phosphorylated and total AMPK, showing representative results of $n=3$ paired independent cell pools (AdGFP, AdCre) and quantification of the signal in $n=6$ pools. Graphs represent mean $\pm \mathrm{SEM}$, and ${ }^{*} P<0.05,{ }^{* *} P<0.01,{ }^{* *} P<0.001$ by Student's $t$ test between genotypes.

caused by Vhl deficiency in osteolineage cells in vivo may therefore affect total glucose uptake in the bones of the mutant mice.

Increased skeletal glucose uptake correlates with reduced glycemia levels. To quantify the glucose consumption by the skeleton, we next performed micro-PET scans and biodistribution assays using the radioactive tracer ${ }^{18} \mathrm{~F}$-fluorodeoxyglucose $\left({ }^{18} \mathrm{~F}\right.$-FDG). In normal adult mice, the skeleton was found to take up a considerable portion of the injected glucose ( $14.7 \%$ of retrieved dose) relative to glu- 

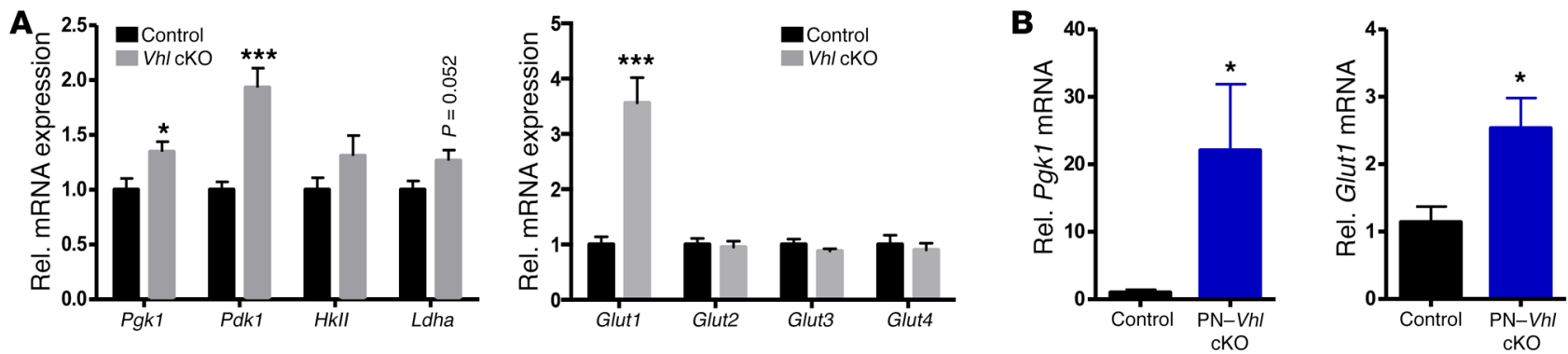

C

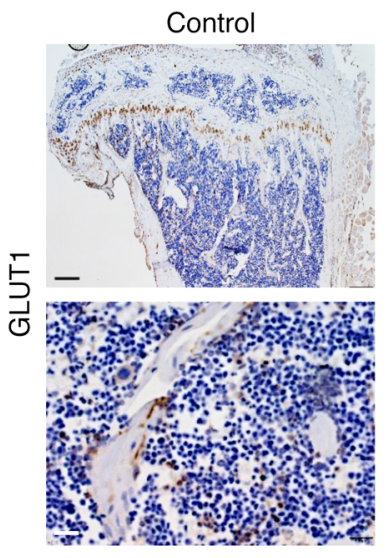

E

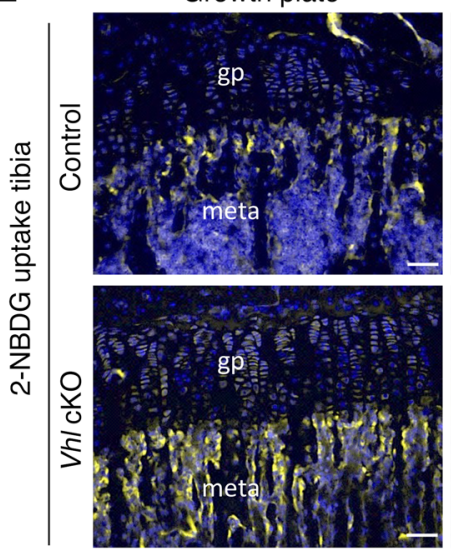

Vhl cKO

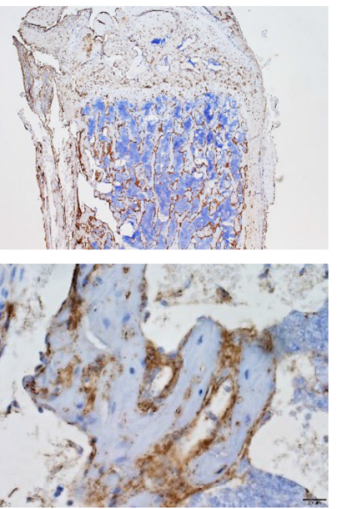

D Control

$\mathrm{PN}-\mathrm{Vhl} \mathrm{CKO}$
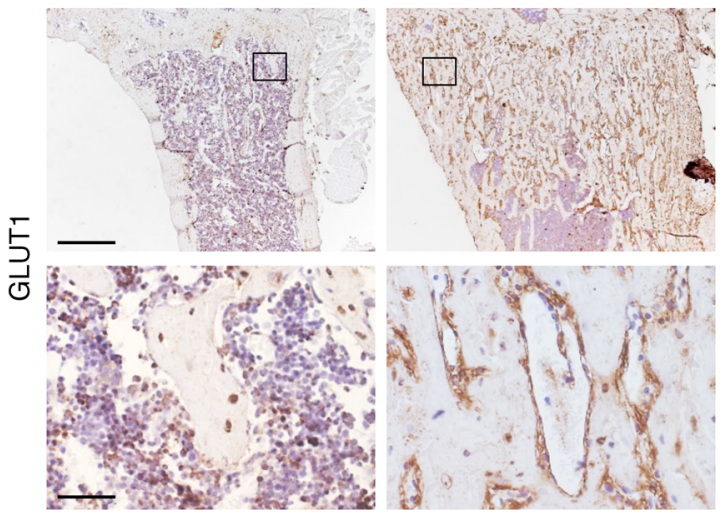

Trabeculae
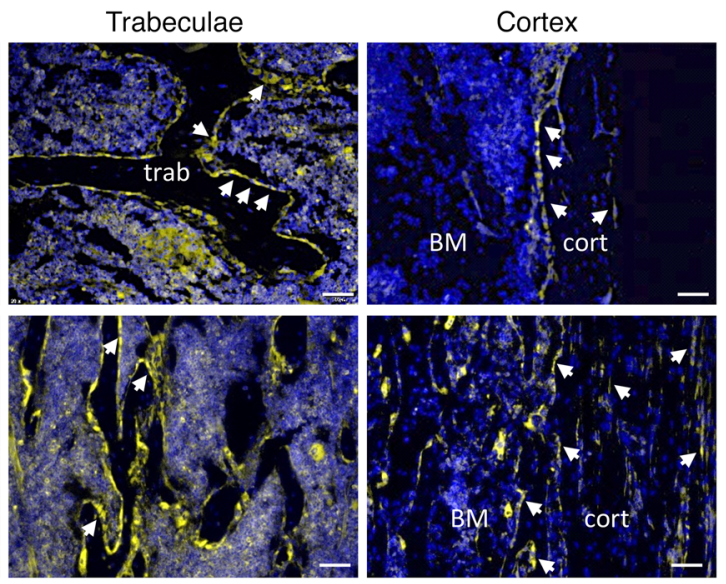

Figure 7. Osteoblast lineage cells are the prime glucose-consuming cells in the skeleton. (A) Relative mRNA levels of $P g k 1, P d k 1, H k I l, L d h a$ (left), and Glut1-4 (right) in bones of 12-week-old control and constitutive VhI cKO mice $(n=7)$. (B) mRNA levels of Pgk1 and Glut1 in bones of control and PN-Vhl cKO mice at 24 weeks ( $n=6-8$ ). (C and D) GLUT-1 IHC on tibia sections of (C) Vhl cKO mice (scale bars: $200 \mu \mathrm{m}$ [top] and $25 \mu \mathrm{m}$ [bottom, magnified views]) and (D) PN-Vhl cKO mice (scale bars: $500 \mu \mathrm{m}$ [top] and $100 \mu \mathrm{m}$ [bottom, magnified views]). (E) Visualization of 2-NBDG uptake in tibias harvested 45 minutes after injection; the signal is strongest in the metaphyseal (meta), trabecular (trab), and cortical (cort) bone regions (most evidently localizing in osteolineage cells on and around the bone surfaces [arrows]), and less intense in growth plate (gp) chondrocytes, BM hematopoietic cells, and bone-embedded osteocytes. Scale bars: $50 \mu \mathrm{m}$. Data are shown as mean \pm SEM; ${ }^{*} P<0.05,{ }^{* *} P<0.001$ by Student's $t$ test. C-E show representative sections of $n=3$ mice analyzed per genotype.

cose storage tissues and high energy-demanding organs (e.g., liver $7.4 \%$, brain $6.3 \%$, heart $11.7 \%$ ) (Figure $8 \mathrm{~A}$ ). Strikingly, the uptake of glucose was significantly and consistently increased in bones (except forelimbs) of $V h l \mathrm{cKO}$ mice compared with control animals, as shown by tissue-specific quantification of the ${ }^{18} \mathrm{~F}-\mathrm{FDG}$ uptake (Figure 8B and Supplemental Table 1 showing soft tissues) and by micro-PET images analyzed over a 60-minute time frame (Figure $8, \mathrm{C}-\mathrm{E}$ ). Time-activity curves of ${ }^{18} \mathrm{~F}$-FDG uptake revealed increased accumulation of the glucose analog in the metaphyseal regions of the tibias and femurs of $\mathrm{Vhl} \mathrm{cKO}$ mice (Figure 8, C-E), correspond- ing to the regions of intense population by glucose-avid osteoblasts (see Figure 7, C-E). Three-compartment model kinetic analyses further substantiated the increased ${ }^{18} \mathrm{~F}$-FDG uptake in the mutant bones (Supplemental Figure 13C). In contrast to the skeleton, which represents the targeted organ in this Osx-Cre:GFP-driven genetic strategy, the liver showed no differences in ${ }^{18} \mathrm{~F}$-FDG uptake between the genotypes (Figure 8, B and F). Most interestingly, we found that the ${ }^{18} \mathrm{~F}$-FDG uptake values in the bones of individual mice negatively correlated with the animal's glycemic levels (Figure 8G), whereas such correlation was not seen in the liver (Figure $8 \mathrm{H}$ ). 

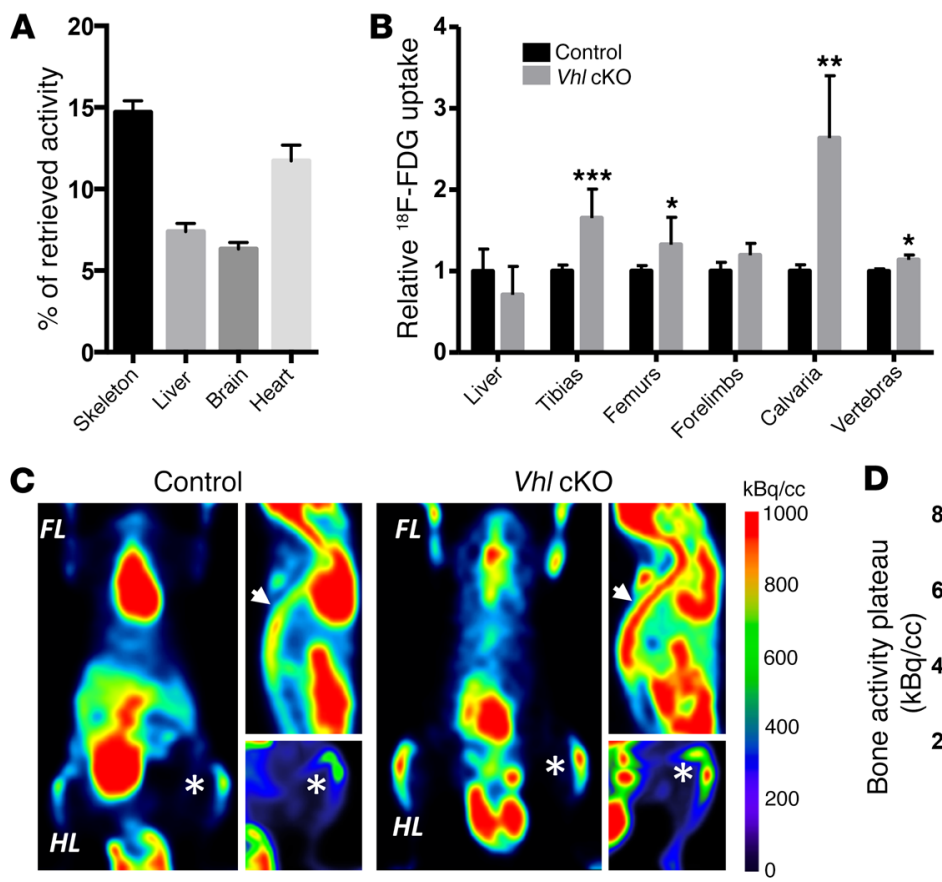

D
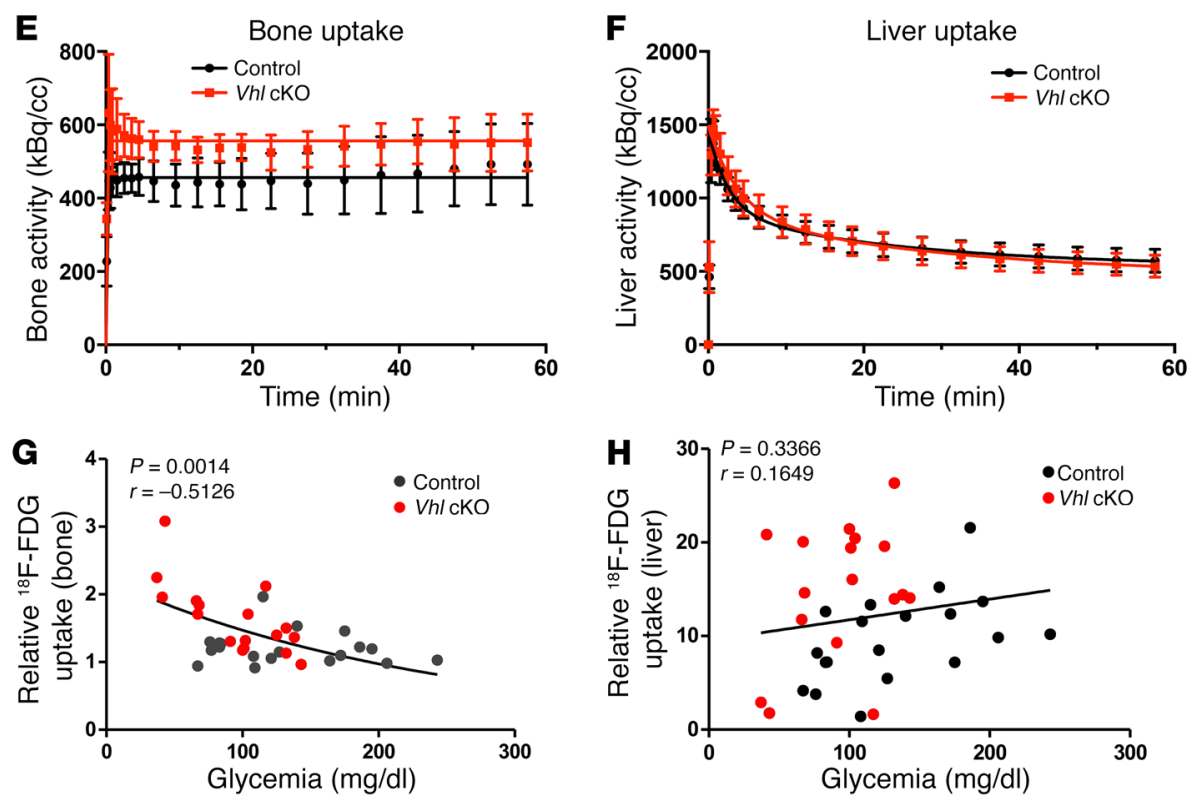

Figure 8. Increased uptake of glucose in bones of $\mathrm{Vhl}$ cKO mice inversely correlates with their blood glucose levels. (A) Uptake of ${ }^{18} \mathrm{~F}-\mathrm{FDC}$ in organs harvested from control mice 45 minutes after i.v. injection, expressed as percentage of the total documented uptake (retrieved activity) in the respective individual animals. Mean \pm SEM, $n=14$. (B) Uptake of ${ }^{18} \mathrm{~F}$-FDG in the liver and various bones of control and $V h /$ cKO mice at 12 weeks, calculated as percentage of the total retrieved activity in the respective animal and expressed relative to the controls ( $n$ $=4-7)$. (C) Time-lapse imaging of ${ }^{18} \mathrm{~F}-\mathrm{FDC}$ uptake by micro-PET ( $n=6-7$ ), showing for each genotype the coronal slice selected for highest ${ }^{18} \mathrm{~F}$-FDG intensity at the level of the tibia (asterisk) (left image), the sagittal slice displaying the highest intensity signal in the vertebral column (arrow) (upper right), and a coronal maximum intensity projection of the hind limb (HL) (lower right). FL, forelimb. (D-F) Kinetic analyses of micro-PET scans over a 60-minute time frame $(n=6-7)$, showing the quantified plateau activity in the distal femur and proximal tibia (D) and kinetic analyses over the full time frame in these bone regions $(\mathbf{E})$ and in the liver $(\mathbf{F})$. ( $\mathbf{C}$ and $\mathbf{H}$ ) Analysis of correlation between glycemia level and relative ${ }^{18} \mathrm{~F}$-FDG uptake (at 45 minutes) in bone (G) or in liver (H). Individual data points of control (black dots) and $\mathrm{Vhl}$ cKO (red dots) mice are shown, and $P$ value and Pearson correlation coefficient $(r)$ values; total $n=36$. Graphs represent mean \pm SEM, and ${ }^{*} P<0.05,{ }^{*} P<0.01$, ${ }^{* *} P<0.001$ by Student's $t$ test between genotypes, unless indicated otherwise.
These findings suggested that manifest uptake of glucose by a selected subset of cells primed toward excessive (HIF-driven) glycolysis, in this case osteoblasts, was able to affect global glucose homeostasis. We next sought to test the conceptual basis of this hypothesis in an independent model and a human setting. Increased glycolysis through activation of the Warburg effect (often even HIF-induced) is a hallmark of malignant cells; moreover, the selective high uptake of glucose by tumor cells forms the basis of the diagnostic use of ${ }^{18} \mathrm{~F}-\mathrm{FDG}$ PET/CT scans for clinical detection of cancer and metastases (15). In questioning whether the activity of glucose-avid tumor cells may be linked to serum glycemia, we analyzed the ${ }^{18} \mathrm{~F}$-FDG PET/CT scans of 10 patients with lung adenocarcinomas (presented in Supplemental Table 2) displaying metastatic cancer lesions in one or several bones (27). Interestingly, the level of glucose uptake in the metastatic lesions (calculated as average maximum and mean ${ }^{18} \mathrm{~F}$-FDG standardized uptake values [SUVs]) inversely correlated with the blood glucose levels and glycated hemoglobin values (Hba1C, a measure of the 3-month average plasma glucose concentration) (Figure 9 and Supplemental Table 3). By contrast, no correlation was found between SUV indices and insulin, HOMA-IR, or HOMA- $\beta$ (denoting $\beta$ cell functioning), or between blood glucose or HbA1c and total osteocalcin or bone resorption, assessed by serum CTX (Supplemental Table 3).

These data support the concept that metabolic rewiring resulting in excessive glucose utilization by a selective subset of cells can effectively impact blood glucose levels, and additionally underscore the potential broad-ranging implications and clinical significance of our findings. 

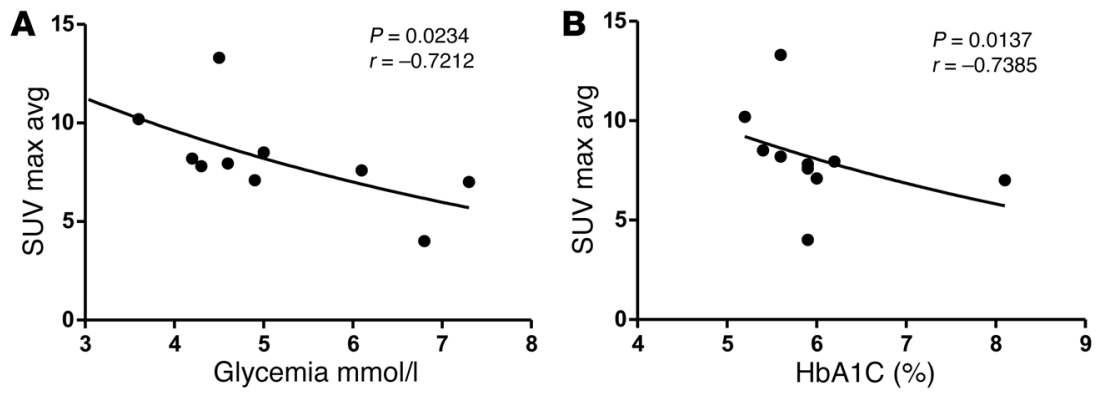

Figure 9. Higher glucose uptake values in glucoseavid tumor metastases correlate with lower glycemia levels in a cohort of patients with lung adenocarcinoma with bone metastases. (A and B) Analysis of correlation between average maximum ${ }^{18} \mathrm{~F}$-FDC SUVs (SUV Max Avg) in bone metastases of individual patients with cancer, and their fasted glycemia levels (A) or glycated hemoglobin (HbA1c) levels (B). Individual patient data points and $P$ value and Spearman correlation coefficient $(r)$ are shown; $n=10$.
Increased glycolysis in Vhl-deficient osteoblast lineage cells can affect systemic glucose homeostasis. The results described above raised the hypothesis that increased glycolysis in osteoblast lineage cells could, possibly directly, lower blood glucose levels and cause disturbances in whole-body glucose homeostasis. If this hypothesis is correct, then pharmacological restoration of the reprogrammed cellular metabolism by administration of a glycolysis inhibitor would be expected to prevent the systemic glucose sensitivity in $V h l$ cKO mice. To test this, we used dichloroacetate (DCA), a compound whose therapeutic benefits against cancer are being tested in clinical trials (15). DCA inhibits glycolysis by inhibiting PDK1, an enzyme that inhibits pyruvate dehydrogenase and thereby the flux of pyruvate into the mitochondria and the tricarboxylic acid cycle (Supplemental Figure 14A). HIF-1 directly and potently induces $P d k 1$ expression in hypoxic cancer cells and conceivably also in our Vhl-deficient osteoblasts (Figure 6C and Figure $7 \mathrm{~A}$ ), thereby promoting the conversion of pyruvate into lactate in the cytosol.

Administration of DCA to control (AdEmpty) and Vhldeficient (AdCre) osteoblasts indeed restored the increased glycolysis (reflected in the ECARs) and the reduced oxidative respiration (as based on the OCRs) of the mutant cells to the baseline control levels (Figure 10A and Supplemental Figure 14B). Consequently, the increased glucose utilization of cells lacking $V h l$ was diminished by DCA in a dose-dependent manner (Supplemental Figure $14 \mathrm{C}$ ). Next, we tested the effect of DCA in vivo (Figure 10B). Interestingly, administration of DCA to $\mathrm{PN}-V h l$ cKO mice prevented the development of the deregulated whole-body glucose metabolism. Specifically, DCA treatment corrected the lower fasted blood glucose levels of $\mathrm{PN}-\mathrm{Vhl} \mathrm{cKO}$ mice (Figure 10C) as well as their increased glucose tolerance (Figure 10D), rendering DCA-treated $\mathrm{PN}-V h l \mathrm{cKO}$ mice globally indistinguishable from their age- and sex-matched control littermates (either vehicleor DCA-treated). No alterations were observed among any of the groups in BW over time or in abdominal fat weight (data not shown). Similar data were obtained in female (Figure 10, C and D) and male (not shown) groups of mice.

In contrast to the rescued systemic metabolic phenotype, bone analyses by micro-CT and histology revealed that the development of the high-bone-mass phenotype in $\mathrm{PN}-V h l$ cKO mice remained unaffected by DCA treatment (Figure 10E and Supplemental Figure 14D). Increased expression of the HIF-target genes Vegf and Epo in bones with conditional Vhl deficiency was also maintained upon DCA treatment (Figure 10F), as were the pronounced local alterations in the bone environment, including the hypervascularization (Supplemental Figure 14D). Likely as a consequence of the partial obliteration of the BM cavity by the excessive bone matrix, $\mathrm{PN}-V h l$ cKO mice showed splenomegaly regardless of the DCA treatment, as documented in both male and female animals (Figure 10G).

These data indicate that pharmacological inhibition of glycolysis by DCA, a compound shown to correct the increased glucose utilization by $V h l$-deficient osteoblasts, was able to prevent the global metabolic phenotype of the skeletal-targeted $V h l \mathrm{cKO}$ mice and uncouple it from the high bone mass. Although DCA was systemically administered, these findings strongly suggest a link between the metabolic rewiring of the mutant osteoblasts toward excessive glycolysis, so-called hyper-Warburgism, and the systemic phenotype of altered glucose homeostasis and energy metabolism (Figure 11).

\section{Discussion}

The data presented here provide genetic and pharmacological support for the concept that the cellular metabolism of the osteoblast and the level of glucose consumption in bone might have repercussions on global glucose clearance and energy homeostasis (Figure 11). These results implicate the hypoxia signaling pathway and local (HIF-driven) glycolysis in the bone-metabolism interplay, and extend our insight into the skeletal contribution to the regulation of integrated, whole-body homeostatic balances.

Hypoglycemia and increased glucose tolerance in skeletal-targeted Vhl mutant mice. While previous studies have shown that activation of the HIF pathway in osteolineage cells is anabolic to bone, to our knowledge no study has previously reported a systemic metabolic phenotype in Osx-Cre-driven Vhl cKO mice or in a comparable mouse model. Yet intriguingly, both the constitutive and the postnatally induced skeletal-targeted $V h l$ cKO mice generated here displayed not only a high bone mass, but also a marked wholebody phenotype characterized by permanently low glycemia levels and increased glucose tolerance. In the constitutive model, the inactivation of $V h l$ early in life led to an increased metabolic rate and failure to build up energy stores, causing a lean appearance with a marked deficiency in body fat. This lipodystrophy or lipoatrophy may plausibly explain the mildly reduced sensitivity to insulin of the constitutive $V h l$ cKO mice; alternatively, it could represent an adaptive response to the permanently low blood glucose levels, in order to prevent hypoglycemic death. Of note, the reduced BW of the constitutive $V h l$ cKO mice was already evident at postnatal week 3 , the time at which doxycycline was only beginning to be washed out of the system in the PN-Vhl cKO model. The postponement of $V h l$ deletion in the Osx-expressing target cell population until 3 weeks of age successfully avoided much of 
A

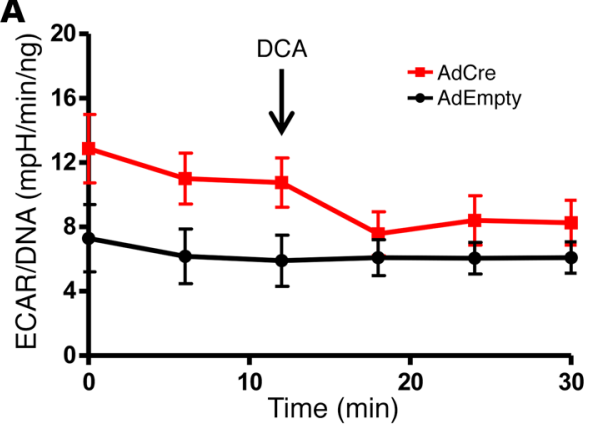

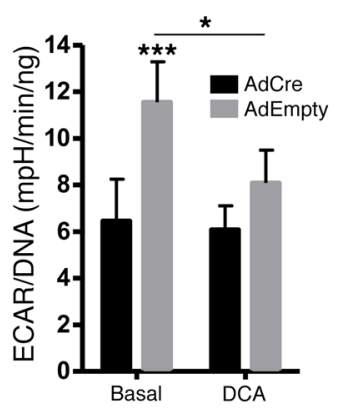

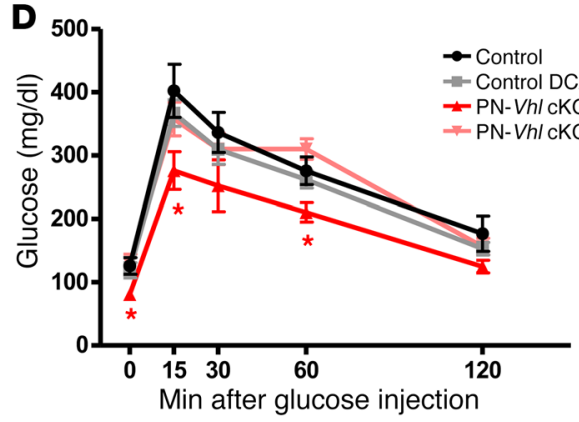

Min after glucose injection
B

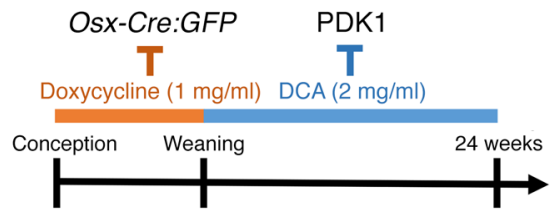

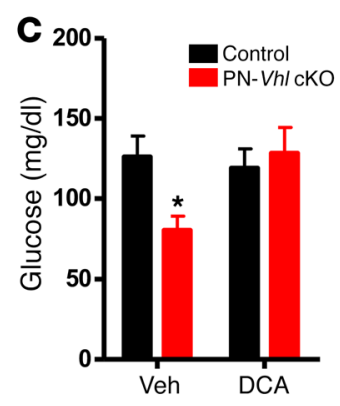

E

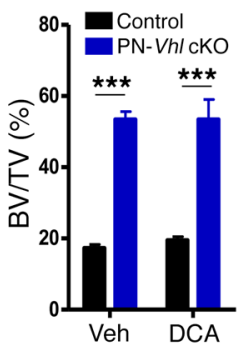

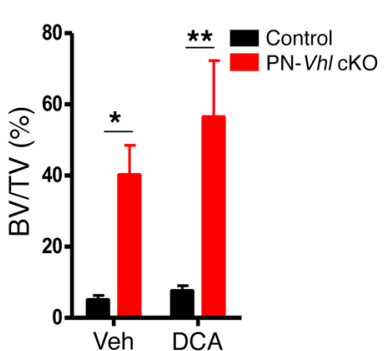
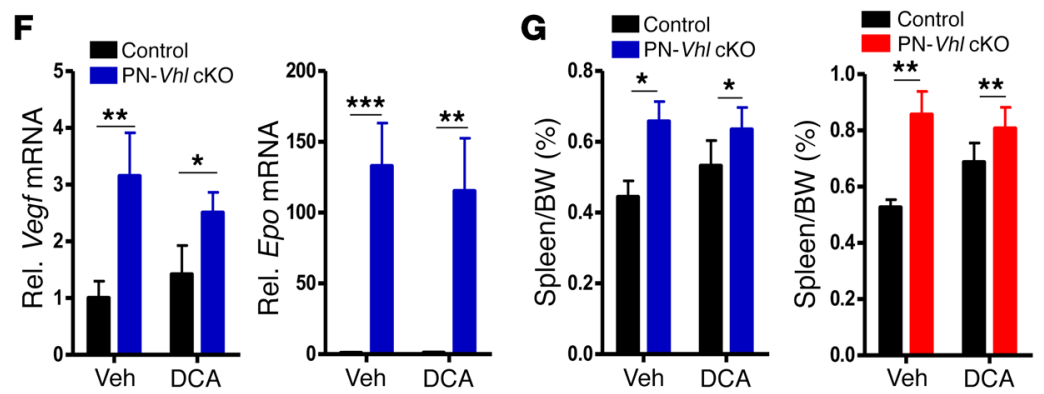

Figure 10. Pharmacological inhibition of glycolysis by DCA specifically prevents the global metabolic phenotype of mice with skeletal Vhl deficiency. (A) ECAR in control (AdEmpty) and Vhl-deficient (AdCre) primary osteoblasts, measured over time (left) and averaged (right) in basal conditions or following administration of DCA at the indicated time $(n=7)$. Data represent mean $\pm \mathrm{SEM}$; $P<0.05$; Student's $t$ test between pairs. (B) Protocol outlining the administration of the PDK1 inhibitor DCA from weeks 3 to 24 of postnatal life of control and PN-Vhl cKO mice, in which Vhl inactivation was postponed by suppressing Osx-Cre:GFP activity with doxycycline. (C) Fasting glucose levels (2-way ANOVA, $P<0.05$ for interaction between genotype and DCA treatment and ${ }^{*} P<0.05$ between control and PN-Vh/ cKO mice in vehicle group by Bonferroni's post test; $n=5-6$ ). (D) GTT ( $P<0.05$; multiple Student's $t$ tests between time points) and corresponding AUC calculations ( 2 -way ANOVA, $P<0.05$ for interaction between genotype and DCA treatment and ${ }^{*} P<$ 0.05 between control and PN-Vhl cKO mice in vehicle group by Bonferroni's post test; $n=5-6)$. (E) Tibia BV/TV determined by ex vivo micro-CT in male ( $n=6-8$; blue bars) and female ( $n=4-5$; red bars) mice. (F) Vegf and Epo mRNA levels in full bones ( $n=7-9$ mice/group). (G) Spleen weight as percentage of BW at 24 weeks in male $(n=6-7)$ and female $(n=5)$ mice. In E-G, graphs represent mean \pm SEM; ${ }^{*} P<0.05$, ${ }^{* *} P<0.01,{ }^{* *} P<0.001 ; 2-$ way ANOVA with Bonferroni's post test. Blue graphs, male data; red graphs, female data.

the potentially confounding phenomena in key endocrine peripheral tissues that contributed to the complexity of the constitutive model (such as the fat deficiency); thus, the $\mathrm{PN}-V h l$ cKO mouse recapitulated the key features of low glycemia and increased glucose tolerance in a context of normal development, early postnatal growth, and baseline physiology. We presume that, by the time of pervasive $V h l$ deletion in the skeleton, the $\mathrm{PN}-V h l$ cKO mice were already relatively resilient to some of the systemic effects of increased glucose uptake by the mutant osteolineage cells, and that their bodies were better furnished than those of the constitutive $V h l \mathrm{cKO}$ mice to respond to these changes and safeguard some of the homeostatic balances. The PN-Vhl cKO model may thereby be viewed as a model of reduced stringency on the system (hypomorph) compared with the effects of constitutive skeletal Vhl loss, which provided us with a simplified and supportive additional model for dissecting how genetic, molecular, and cellular changes in the bone environment can affect systemic glucose metabolism.

Initially, we hypothesized that osteocalcin may have been involved in integrating the skeletal mutagenesis with the global repercussions on glucose homeostasis and energy metabolism. However, the unexpectedly low production and strikingly low circulating levels of osteocalcin in the Vhl-deficiency models (that reflect impaired terminal osteoblast differentiation and low bone turnover, as discussed below) are not in correspondence with a dominant role for osteocalcin signaling in the systemic phenotype of our mice. The serum levels of osteocalcin (total and undercarboxylated forms) are generally inversely related to plasma glucose, fat mass, and the degree of insulin resistance in mice and 


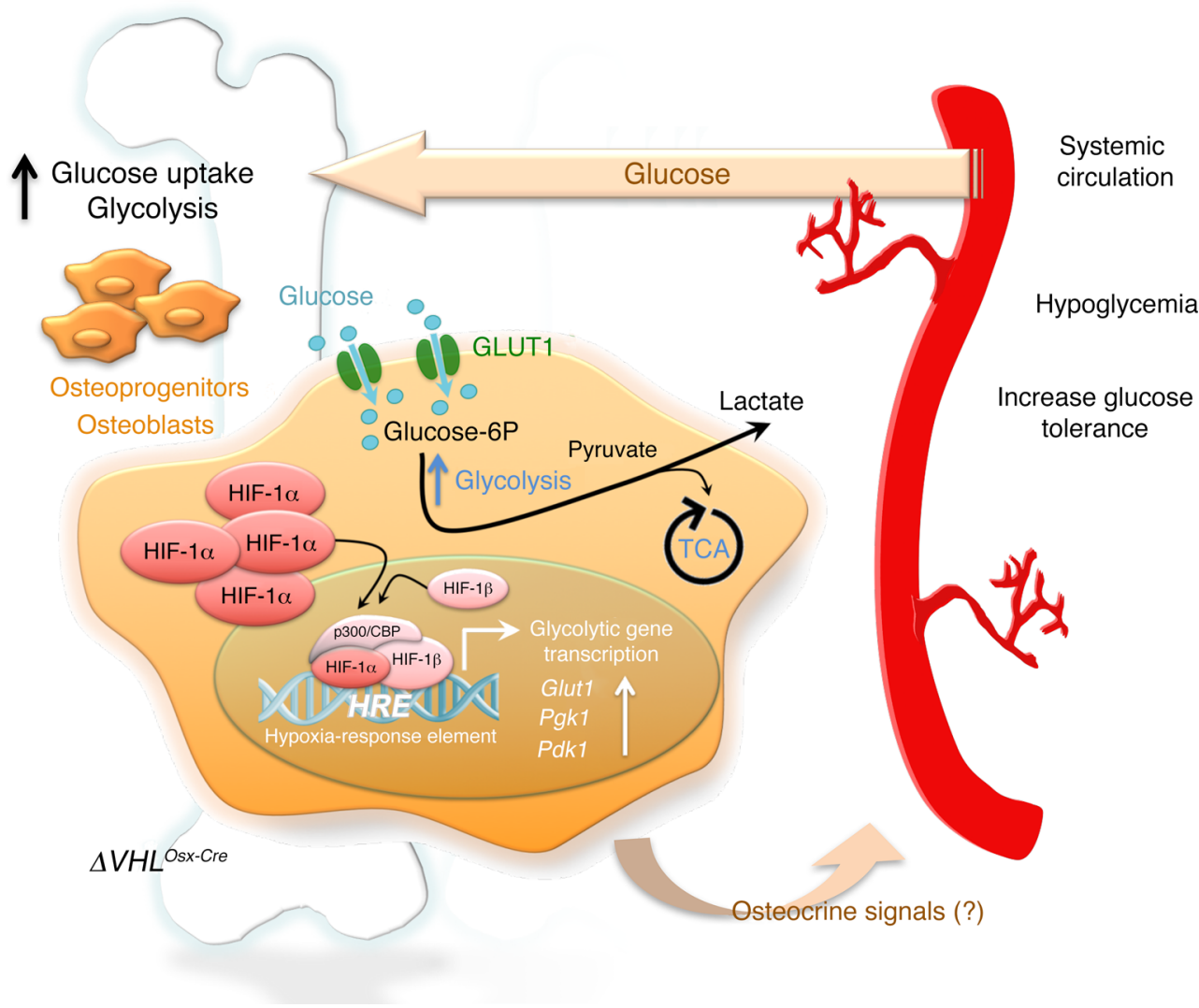

Figure 11. Schematic summary. Left: $V h l$ inactivation in osteoprogenitors and the osteoblast lineage cells derived from them locally leads to excessive HIF stabilization and transcriptional activity, including strong upregulation of glucose transporters (Glut1) and glycolysis-promoting enzymes (Pgk1, $P d k 1)$. These molecular changes are associated with increased glucose uptake and glycolysis in the Vhl-deficient osteolineage cells, and increased glucose consumption by the skeleton as a whole. Right: Systemically, Vhl cKO mice showed consistently reduced blood glucose levels and an increased glucose tolerance that could not be explained through effects on insulin or osteocalcin. Link between local and systemic phenotypes: Since systemic administration of the glycolysis inhibitor DCA rescued the metabolic phenotype, it is possible that the low glycemia was a direct consequence of the increased uptake of glucose in bone, although potential contributions by unknown endocrine-acting osteokines (osteocrine signals) cannot at present be excluded. These new findings strongly suggest that local glucose utilization in the skeleton contributes to systemic glucose clearance and metabolic homeostasis, a concept that may help in the development of therapies beneficial for both bone and metabolic health.

humans $(3,28,29)$. Osteocalcin ${ }^{-/}$mice show an obese phenotype with hyperglycemia, hypoinsulinemia, and reduced insulin secretion and sensitivity compared with WT mice (3). The reduced insulin sensitivity of the constitutive $V h l$ cKO mice may thus possibly be explained through the endocrine actions of osteocalcin, but certainly not their hypoglycemic and lean phenotype. In fact, the low blood glucose levels in both our $\mathrm{Vhl}$-deficient mouse models would be expected to lower insulin production and increase insulin sensitivity; yet these responses were not seen, suggesting that counteracting mechanisms may have been operating to increase insulin production and reduce insulin sensitivity. Low osteocalcin would be expected to reduce insulin sensitivity (3), which could reflect a contribution of the low osteocalcin levels to the global metabolic phenotypes. With regard to the insulin levels, however, low osteocalcin cannot provide such a counteracting mechanism, as low osteocalcin is itself associated with reduced insulin production and secretion. The reasons underlying the aberrantly normal insulin levels in these hypoglycemic mice thus remain enigmatic.

Given that the alterations in global glucose homeostasis in mice with $V h l$ inactivation could not be simply explained through the known endocrine actions of insulin or osteocalcin, our find- ings support the notion that osteolineage cells can impact global energy metabolism through other as-yet-uncharacterized mechanisms, as suggested previously (6-8).

Cellular glucose utilization by osteoblasts as a determinant of global energy homeostasis. Intriguingly, while the low blood glucose levels in Vhl-depleted mice could not be explained by increased production of or sensitivity to insulin, nor by increased levels of osteocalcin, they instead correlated significantly with increased skeletal uptake of glucose from the circulation, as evidenced by in vivo ${ }^{18} \mathrm{~F}-\mathrm{FDG}$ tracer experiments. While glucose uptake was overall increased in bones of the mutant mice, ${ }^{18} \mathrm{~F}-\mathrm{FDG}$ levels were normal in the majority of soft tissues (such as liver, spleen, lungs, heart, stomach, and skeletal muscle, as determined in resting conditions, under general anesthesia [see Supplemental Table 1]). Strikingly, already in a basal (WT) setting, the skeleton presented as a major contributor to the body's glucose consumption, accounting for almost $15 \%$ of the glucose uptake from the blood in anesthetized mice. This uptake is similar to or even higher than that seen in some of the established glucose-avid tissues such as heart, liver, and brain, a finding that is in line with other recent work (30). Plausible explanations are the large total contribution of the skel- 
eton to the body and the high energetic demands associated with bone formation and maintenance. In accordance with this latter notion, we found that within bone, the most marked glucose-avid cell types were those residing on and in proximity of the trabecular and cortical bone surfaces, corresponding to osteoprogenitors and osteoblasts by location and morphology. Osteocytes appeared to contribute little to glucose consumption in murine bones.

Osteolineage cells thus appear responsible for a great deal of the skeletal glucose consumption. Early in vitro work indicated that osteogenic cells are highly glycolytic $(31,32)$. Recent in vivo studies provided evidence that glucose uptake in osteoblasts stands partly under the control of insulin, is mediated via GLUT1 and GLUT4, and is crucial for osteoblast differentiation and bone formation $(18,19)$. Our data now provide in vivo evidence that increased utilization of glucose by osteoblasts, such as instigated by genetic activation of HIF signaling, can put substantial pressure on global glucose homeostasis. Notably, the number of osteolineage cells in the body, taken cumulatively in the more than 200 bones constituting the skeleton, is likely considerable. In this light, it seems plausible that drastic changes in their glucose consumption could have repercussions on overall systemic glucose homeostasis. Vhl deletion greatly increased the glycolytic flux and glucose utilization by osteogenic cells in vitro and in vivo. Such a shift in the use of glucose as a source of energy from oxidative phosphorylation toward its breakdown via glycolysis, accompanied by increased glucose uptake to maintain energy demands (ATP production), resembles the Warburg effect that typifies cancer cells (13-15). Interestingly, the experimental anticancer drug and glycolysis inhibitor DCA corrected, at least partly, the bioenergetic switch and increased glucose consumption of $V h l$-deficient osteolineage cells, and prevented the global metabolic phenotype. Thus, osteolineage cells are largely responsible for the high skeletal demand of glucose, and inducing a state of hyper-Warburgism in these cells can increase the local glycolytic flux and glucose utilization, apparently even to such an extent that it affects the overall glucose clearance from the circulation (Figure 11).

It thus seems that a continual drain of glucose toward the skeleton (as is possibly the case for other tissues) is able to cause an uncompensated imbalance in global glucose homeostasis, leading to an altered overall energy metabolism and even a failure to build up or a depletion of the body's energy stores. The potential farreaching therapeutic implications of this concept are reinforced by our finding that patients carrying glucose-avid bone metastatic tumor masses also showed an inverse correlation between the local glucose uptake in the cancer lesions and global glycemia levels. This observation opens an avenue of potential significance in tumor diagnosis, management, and treatment, warranting further investigation. Notable in this regard, for instance, is the rare but documented occurrence of clinically asymptomatic hypoglycemia and lactic acidosis in certain nonpancreatic malignancies, especially lymphomas, which has been attributed to an extreme manifestation of the Warburg effect (33-35).

Possibly, a rewired osteoblastic metabolism may be able to influence systemic glucose clearance directly, although involvement of secondary osteocrine signals cannot be excluded (Figure 11). Yet the finding that DCA could uncouple the high-bone-mass phenotype from the increased glucose tolerance in $\mathrm{PN}-V h l \mathrm{cKO}$ mice, by rescuing specifically the whole-body metabolic imbalance while having no discernible impact on the local bone manifestations, may argue against a critical role of endocrine signals emanating from the anomalous bone but in favor of a direct mechanism. Possibly in line with this hypothesis, mice overexpressing GLUT1 in osteoblasts showed systemic metabolic alterations similarly including increased glucose tolerance, although high circulating osteocalcin levels could also explain the phenotype in this model (18). This may also predict that, conversely, a significant reduction in osteoblast number or a deficiency in osteoblastic glucose uptake may be associated with reduced skeletal glucose utilization and impaired systemic glucose disposal. In this regard, it is interesting to note that mouse models of osteoblast ablation or of osteoblast-specific deletion of GLUT1 or GLUT4 were all associated with reduced glucose clearance and/or hyperglycemia, although these phenotypes were complex and often attributable at least in part to osteocalcin $(6,18,19)$.

Combined VHL/HIF downstream effectors mediate the highbone-mass phenotype in mice lacking Vhl in osteolineage cells. Our data indicate that skeletal-specific $V h l \mathrm{cKO}$ mice have high bone mass and a disrupted BM microenvironment that is heavily vascularized with dilated blood vessels. Surprisingly, the bone formation and mineralization rate was greatly decreased at 12 weeks of age, associated with impaired terminal differentiation of osteoblasts and an expansion of the pool of relatively immature osteolineage cells depositing disorganized, woven bone matrix. No major alterations could be detected in the number of osteoclasts; however, the presence of aberrant cartilage remnants within the trabecular bone strongly suggests impaired osteoclast functioning. Inefficient resorption of the matrix could be caused by osteoclastic alterations or, alternatively, by anomalous modifications of the matrix rendering it more resistant to degradation. The latter could result, for instance, from alterations in the biosynthesis of collagens in chondrocytes and osteolineage cells, as HIF signaling is known to improve the efficiency of posttranslational hydroxylation of collagens and collagen crosslinking, thereby determining the conformational stability of collagen triple helices $(10,36,37)$. The low bone turnover state at adult ages in $V h l$-deficient mice indicates that increased bone matrix deposition and mineralization must have occurred at earlier stages in life, similar to the findings made upon Ocn-Cre-driven $V h l$ inactivation, targeting mature osteoblasts (11). These mice showed increased bone formation at 7 days of age, followed by a decline in bone turnover associated with progressively increased bone mass (11).

Intriguingly, DCA treatment revealed that blocking glycolysis only reversed the low glycemia and increased glucose tolerance, but not the augmented bone mass of $V h l$ mutants. In other words, the local effect on bone mass and the systemic effects on energy metabolism could be uncoupled by DCA-mediated inhibition of glycolysis. This finding indicates that, on the one hand, the control of glucose homeostasis by osteoblast lineage cells is not a bona fide consequence of the high bone mass (as discussed above), and on the other hand, the high bone mass does not rely solely on the enhanced glycolysis. This latter aspect can be reconciled with the findings by Regan et al. (21), who found that DCA did restore the increased bone mass of mice conditionally expressing a stabilized form of HIF- $1 \alpha$, by the fact that $V h l$ deletion also stabilizes HIF- $2 \alpha$, 
as well as other less-well-characterized factors (26). The repercussions of enhanced signaling by HIF- $1 \alpha$ versus HIF- $2 \alpha$ were recently studied in models of Os $x$-Cre:GFP-driven overexpression of the respective HIFs (38), confirming and extending earlier studies indicating that the genes regulated by HIF- $1 \alpha$ and HIF- $2 \alpha$ in osteoblasts are overlapping but nonidentical. While HIF-1 $\alpha$ appears to be primarily responsible for meditating the metabolic switch to glycolysis, VEGF upregulation in osteogenic cells is controlled by both HIF- $1 \alpha$ and HIF- $2 \alpha(10,21,38)$. Additionally, HIF- $2 \alpha$ has been shown to be the main regulator for EPO production by osteoblasts (12) and to regulate OPG, the factor that inhibits osteoclastogenesis by counteracting RANKL (38). All of these downstream effectors of the VHL/HIF axis - whose increased expression fully correlated with the presence of the local bone phenotype in our Vhl-deficient models, including in being unaffected by DCA treatment - could have contributed to or caused the high bone mass and BM alterations. First, VEGF represents a key player in the tight coupling of angiogenesis and osteogenesis. Several studies have shown that inhibiting or increasing angiogenesis by modulation of VEGF expression decreases or stimulates bone formation, respectively $(10,39-41)$. Overall, the bone phenotype described here in $\mathrm{Vhl}$ cKO mice resembles in many respects the phenotype associated with induced VEGF overexpression in the osteochondrolineage cells of adult mouse bones, including the observed hypervascularization, BM fibrosis, and unbalanced bone formation and turnover (41). This suggests that increased VEGF expression by VHL-deficient osteoblasts, and consequent VEGF-mediated angiogenic-osteogenic coupling, constitutes a prime contributor to the local bone alterations in $V h l \mathrm{cKO}$ mice. Second, besides regulating erythropoiesis, EPO has also been shown to stimulate bone formation and repair (42). Third, OPG has been recognized as a direct transcriptional target of HIF- $2 \alpha$, and at least part of the net bone anabolic effect of HIF- $2 \alpha$ has been ascribed to reduced bone resorption (38).

Altogether, our data on 2 models of $V h l$ deletion, in light of the available knowledge and published work, suggest that the changes in systemic energy metabolism are a consequence of increased osteolineage cell glycolysis and bone glucose uptake, whereas the high bone mass is presumably due to a combination of mechanisms, including - possibly among others - increased skeletal vascularization and altered angiogenic-osteogenic coupling, excessive glycolytic pathway activation in osteolineage cells, increased activity of early osteolineage cells with unbalanced bone formation and mineralization, and reduced bone resorption. These combined effects are mediated by dysregulation of various genes downstream of both HIF- $1 \alpha$ and HIF- $2 \alpha$.

In conclusion, this study reveals that cellular glucose utilization by osteoblasts may represent an important determinant of global glucose homeostasis, with the capacity to override the established endocrine mechanisms involving the osteocalcin-insulin axis. This new link between bone and systemic energy metabolism may be direct, or act via as-yet-uncharacterized osteocrine factors, and stands under the control of the hypoxia signaling pathway in osteolineage cells (Figure 11). These findings may have implications for the future use of bone anabolic therapies for osteoporosis, particularly regarding their potential interplay with metabolic homeostasis and disorders such as obesity and diabetes mellitus.

\section{Methods}

Animals. Osx-Cre:GFP mice (24) were crossed with Vhl floxed mice (25) in a mixed genetic background, as detailed in Supplemental Methods. For suppression of $V h l$ inactivation, doxycycline (Sigma-Aldrich) was added to the drinking water at $1 \mathrm{mg} / \mathrm{ml}$ and refreshed 3 times a week from conception until P21. Next, mice were randomly assigned to DCA (Sigma-Aldrich) or vehicle treatment groups; DCA was administered from 3 until 24 weeks of age $(2 \mathrm{mg} / \mathrm{ml}$ drinking water).

Micro-CT. We used the in vivo Skyscan 1076 micro-CT system (Bruker) with scanning parameters $50 \mathrm{kV}, 100 \mu \mathrm{A}$, and 9- $\mu \mathrm{m}$ voxel size, and applied a 1-mm aluminum filter. Postmortem micro-CT scans of tibias and vertebrae were made using the ex vivo Skyscan 1172 device (Bruker) at $50 \mathrm{kV}, 200 \mu \mathrm{A}, 5-\mu \mathrm{m}$ voxel size, and a $0.5-\mathrm{mm}$ aluminum filter. Scans were reconstructed and analyzed using NRecon, CTAn, and CTVol software (Bruker), according to standardized protocols as detailed in Supplemental Methods.

Histology, IHC, and histomorphometry. Bones and soft tissues were processed for histology and stained as previously described $(40,41)$. IHC for PECAM-1/CD31 (BD Biosciences, catalog 550274) on paraffin sections was as previously described (43). For costaining with GFP antibodies (chicken anti-GFP, $10 \mu \mathrm{g} / \mathrm{ml}$, Abcam, ab13970), frozen sections were used; the PECAM-1 signal was amplified using fluoresceintyramide according to the TSA Cyanine 3 System (PerkinElmer) and GFP was detected using a secondary goat anti-chicken antibody (5 $\mu \mathrm{g} / \mathrm{ml}$, Abcam, ab96951). Paraffin sections were reacted for periodic acid-Schiff (PAS) or reticulin using commercial kits (Sigma-Aldrich). For GLUT-1 IHC, we used rabbit anti-mouse primary antibodies (MilliporeSigma, 07-1401) at $2.5 \mu \mathrm{g} / \mathrm{ml}$ and goat-anti-rabbit polyclonal secondary antibodies (Abcam, ab6720) at $5 \mu \mathrm{g} / \mathrm{ml}$. Insulin IHC was performed as described in Supplemental Methods. Images were taken with an Olympus IX83 inverted microscope equipped with DP73 camera. Histomorphometry was performed as previously described (44) and as detailed in Supplemental Methods.

Cell culture studies. Primary osteoblasts were isolated from long bones of 12-week-old $V h t^{t / f l}$ mice by enzymatic digestion as outlined in Supplemental Methods, combining the yield from 3 mice to obtain 1 independent cell pool. Each cell pool was split into a pair of an experimental and a control sample, transduced respectively with adenoviruses encoding Cre (AdCre) or carrying a GFP-expressing or empty vector (AdGFP/AdEmpty; Viral Vector Core, University of Iowa; see Supplemental Methods). Glucose and lactate levels were measured in the conditioned medium. Glucose uptake in the cells was determined by adding 2-( $N$-(7-nitrobenz-2-oxa-1,3-diazol-4-yl)amino)-2-deoxyglucose (2-NBDG; ThermoFisher Scientific) to the medium at $100 \mu \mathrm{M}$ for 8 hours and measuring fluorescence at $485 / 540 \mathrm{~nm}$. ATP production was quantified using the ATPLite kit (PerkinElmer). Extracellular flux analysis was performed on a Seahorse Bioscience XFp analyzer (Agilent), measuring OCR and ECAR during baseline conditions and after addition of mitochondrial stress test components $(1 \mu \mathrm{M}$ oligomycin, $1 \mu \mathrm{M}$ FCCP, $0.5 \mu \mathrm{M}$ rotenone) or DCA (50 mM; MilliporeSigma). Results were normalized for DNA content of the corresponding culture well. In vitro osteogenic differentiation was performed as previously described (44).

Gene expression analysis. Western blot and real-time quantitative RT-PCR were performed as described in Supplemental Methods, using the primers and probes detailed in Supplemental Table 4. Relative mRNA levels were calculated by the $\Delta \Delta \mathrm{Ct}$ method, using Hprt as housekeeping gene. 
Metabolic studies and bioassays. For GTT and GSIS, glucose was injected i.p. after overnight fasting, at $2 \mathrm{~g} / \mathrm{kg}$ BW and $3 \mathrm{~g} / \mathrm{kg} \mathrm{BW}$, respectively. For ITT, mice were fasted for 3 hours and injected i.p. with $0.75 \mathrm{U} / \mathrm{kg}$ BW insulin. Blood glucose was measured using a OneTouch Verio glucose monitor (LifeScan). ELISA assays were used to determine serum insulin, leptin, adiponectin (all from Crystal Chem), osteocalcin (Immunotopics), CTX, and P1NP (both from ImmunoDiagnostic Systems). Indirect calorimetry was done as described in Supplemental Methods.

Radioactive glucose tracing. ${ }^{18} \mathrm{~F}-\mathrm{FDG}$ biodistribution assays and micro-PET imaging were performed in the KU Leuven molecular Small Animal Imaging Centre (moSAIC). ${ }^{18} \mathrm{~F}-\mathrm{FDG}$ was prepared through an Ion Beam Applications synthesis module. After overnight fasting, 10- to 12-week-old mice were anesthetized with isoflurane inhalation before tail vein injection with ${ }^{18} \mathrm{~F}-\mathrm{FDG}$ (doses given in $\mu \mathrm{Ci}=$ $\mathrm{BW} \times 16$ ). Mice were sacrificed 45 minutes later, or subjected to smallanimal PET imaging for 60 minutes using a lutetium oxyorthosilicate detector-based FOCUS 220 tomograph (Siemens/Concorde Microsystems). Time-activity curves were made using PMODv.3.1 software (PMOD Technologies LLC). For details, see Supplemental Methods.

Fluorescent glucose tracing experiments. Eight-week-old anesthetized mice were injected with $25 \mathrm{mg} / \mathrm{kg}$ BW of 2-NBDG via the tail vein, and sacrificed 5 or 45 minutes after injection. Tissues were fixed with $4 \%$ paraformaldehyde at $4^{\circ} \mathrm{C}$ for 4 hours. Calvaria were imaged whole-mount using a MZ165 stereomicroscope (Leica). Tibias were decalcified for 2 weeks in 0.5 M EDTA, embedded in freezing medium (Freeze Gel Q Path, VWR), sectioned, and analyzed and imaged using an Olympus IX83 microscope.

Human data. We investigated data from 10 patients from the noninterventional, prospective POUMOS-TEC cohort that includes patients presenting with first bone metastases from adenocarcinoma lung cancer (stage IV) (27). As detailed in Supplemental Methods, serum biochemical parameters and FDG SUV parameters for each bone metastatic lesion observed by ${ }^{18} \mathrm{~F}-\mathrm{FDG}$ PET/CT scans were determined. Correlations were computed and assessed using 2-tailed nonparametric tests.

Statistics. All data are presented as mean \pm SEM. Comparisons between 2 groups were done by 2-tailed Student's $t$ tests. For multiple comparisons between groups, we performed 2-way ANOVA with Bonferroni post hoc tests (GraphPad Prism 5). Correlations were computed and assessed using Pearson correlation coefficient tests. For the human data, correlations were computed by nonparametric Spearman correlation coefficient tests. Throughout the study, $P$ values below 0.05 were considered significant.
Study approval. The animal experiments were in accordance with the institutional authorities' guidelines and formally approved by the Animal Ethics Committee of the KU Leuven. The POUMOS-TEC cohort was approved by the local ethics committee (CPP Sud Est IV, Lyon, France) and registered into ClinicalTrials.gov under the ID NCT02810262. Patients provided written informed consent.

\section{Author contributions}

ND, TLC, and C. Maes designed the study. ND performed the majority of the experiments, with specific contributions by RJT, EMM, RV, and CMT, and assistance from TB, EN, and RC. C. Mathieu, BVDS, $\mathrm{CBC}$, and TLC contributed essential equipment, expertise, clinical data, and critical suggestions. ND and C. Maes analyzed data and wrote the manuscript. C. Maes supervised the study.

\section{Acknowledgments}

The authors thank R. Kroes, B. Dubois, M.C. Carlier, and L. Chambard for assistance; A.M. Böhm and M. Mesnieres for help in optimization of techniques; A. Van Santvoort, T. Buelens, C. Casteels, J. Wouters, B. de Laat, and K. Van Laere for sharing small-animal imaging infrastructure and help with tracer assays; and P. Agostinis, A. van Vliet, and S. Van Eygen for Seahorse use. We thank A. McMahon and E. Schipani for sharing mouse lines. T.J. Martin, H. Kronenberg, and F. Luyten are acknowledged for critically reading the manuscript and providing valuable input. We also thank F. Giammarile, P. Clézardin, N. Girard, and the members of the SBE for helpful discussions. This work was supported by grants from the European Research Council (ERC Starting Grant 282131 under the European Union's Seventh Framework Programme, FP/2007-2013), Research Foundation Flanders (FWO, G.094416N), and University of Leuven (OT/14/121) to C. Maes, and by the NIH/National Institute of Arthritis and Musculoskeletal and Skin Diseases (subaward to C. Maes of R01AR049410 to TLC). CBC is supported by the Hospices Civils de Lyon (Young Investigator Grant 2011), EMM is an FWO fellow, and ND holds a doctoral fellowship of the Agency for Innovation by Science and Technology in Flanders (IWT).

Address correspondence to: Christa Maes, Laboratory of Skeletal Cell Biology and Physiology (SCEBP), Skeletal Biology and Engineering Research Center (SBE), Department of Development and Regeneration, KU Leuven, Gasthuisberg O\&N 1, Herestraat 49, Box 813, B-3000 Leuven, Belgium. Phone: 32.16.37.26.56; Email: christa.maes@kuleuven.be.
1. Karsenty G, Ferron M. The contribution of bone to whole-organism physiology. Nature. 2012;481(7381):314-320.

2. Ducy P. The role of osteocalcin in the endocrine cross-talk between bone remodelling and energy metabolism. Diabetologia. 2011;54(6):1291-1297.

3. Lee NK, et al. Endocrine regulation of energy metabolism by the skeleton. Cell. 2007;130(3):456-469.

4. Fulzele K, et al. Insulin receptor signaling in osteoblasts regulates postnatal bone acquisition and body composition. Cell. 2010;142(2):309-319.

5. Ferron $\mathrm{M}$, et al. Insulin signaling in osteoblasts integrates bone remodeling and energy metabolism. Cell. 2010;142(2):296-308.

6. Yoshikawa Y, et al. Genetic evidence points to an osteocalcin-independent influence of osteoblasts on energy metabolism. J Bone Miner Res. 2011;26(9):2012-2025.

7. Gillespie JR, et al. GSK-3 $\beta$ function in bone regulates skeletal development, whole-body metabolism, and male life span. Endocrinology. 2013;154(10):3702-3718.

8. Yao Q, Yu C, Zhang X, Zhang K, Guo J, Song L. Wnt/ $\beta$-catenin signaling in osteoblasts regulates global energy metabolism. Bone. 2017;97:175-183.
9. Semenza GL. Hypoxia-inducible factors in physiology and medicine. Cell. 2012;148(3):399-408.

10. Maes C, Carmeliet G, Schipani E. Hypoxia-driven pathways in bone development, regeneration and disease. Nat Rev Rheumatol. 2012;8(6):358-366.

11. Wang Y, et al. The hypoxia-inducible factor alpha pathway couples angiogenesis to osteogenesis during skeletal development. J Clin Invest. 2007;117(6):1616-1626.

12. Rankin EB, et al. The HIF signaling pathway in osteoblasts directly modulates erythropoiesis through the production of EPO. Cell. 2012;149(1):63-74. 
13. Warburg O. On the origin of cancer cells. Science. 1956;123(3191):309-314.

14. Hanahan D, Weinberg RA. Hallmarks of cancer: the next generation. Cell. 2011;144(5):646-674.

15. Vander Heiden MG. Targeting cancer metabolism: a therapeutic window opens. Nat Rev Drug Discov. 2011;10(9):671-684.

16. Guntur AR, Le PT, Farber CR, Rosen CJ. Bioenergetics during calvarial osteoblast differentiation reflect strain differences in bone mass. Endocrinology. 2014;155(5):1589-1595.

17. Lee WC, Guntur AR, Long F, Rosen CJ. Energy metabolism of the osteoblast: implications for osteoporosis. Endocr Rev. 2017;38(3):255-266.

18. Wei J, et al. Glucose uptake and Runx2 synergize to orchestrate osteoblast differentiation and bone formation. Cell. 2015;161(7):1576-1591.

19. Li Z, et al. Glucose transporter-4 facilitates insulin-stimulated glucose uptake in osteoblasts. Endocrinology. 2016;157(11):4094-4103.

20. Esen E, Chen J, Karner CM, Okunade AL, Patterson BW, Long F. WNT-LRP5 signaling induces Warburg effect through mTORC2 activation during osteoblast differentiation. Cell Metab. 2013;17(5):745-755.

21. Regan JN, et al. Up-regulation of glycolytic metabolism is required for HIF1 $\alpha$-driven bone formation. Proc Natl Acad Sci U S A. 2014;111(23):8673-8678.

22. Esen E, Lee SY, Wice BM, Long F. PTH promotes bone anabolism by stimulating aerobic glycolysis via IGF signaling. J Bone Miner Res. 2015;30(11):1959-1968.

23. Karner CM, Esen E, Chen J, Hsu FF, Turk J, Long F. Wnt Protein signaling reduces nuclear Acetyl-CoA levels to suppress gene expression during osteoblast differentiation. J Biol Chem. 2016;291(25):13028-13039.

24. Rodda SJ, McMahon AP. Distinct roles for Hedgehog and canonical Wnt signaling in specification, differentiation and maintenance of osteoblast progenitors. Development. 2006;133(16):3231-3244.

25. Haase VH, Glickman JN, Socolovsky M, Jaenisch R. Vascular tumors in livers with targeted inactivation of the von Hippel-Lindau tumor suppressor Proc Natl Acad Sci U S A. 2001;98(4):1583-1588.

26. Hsu T. Complex cellular functions of the von Hippel-Lindau tumor suppressor gene: insights from model organisms. Oncogene. 2012;31(18):2247-2257.

27. Confavreux CB, et al. Mutational profiling of bone metastases from lung adenocarcinoma results of a prospective study (POUMOS-TEC). Bonekey Rep. 2014;3:580.

28. Kindblom JM, et al. Plasma osteocalcin is inversely related to fat mass and plasma glucose in elderly Swedish men. J Bone Miner Res. 2009;24(5):785-791.

29. Confavreux CB, Szulc P, Casey R, Varennes A, Goudable J, Chapurlat RD. Lower serum osteocalcin is associated with more severe metabolic syndrome in elderly men from the MINOS cohort. Eur J Endocrinol. 2014;171(2):275-283.

30. Zoch ML, Abou DS, Clemens TL, Thorek DL, Riddle RC. In vivo radiometric analysis of glucose uptake and distribution in mouse bone. Bone Res. 2016;4:16004.

31. Borle AB, Nichols $N$, Nichols G. Metabolic studies of bone in vitro. I. Normal bone. J Biol Chem. 1960;235:1206-1210.

32. Felix R, Neuman WF, Fleisch H. Aerobic glycolysis in bone: lactic acid production by rat calvaria cells in culture. Am J Physiol. 1978;234(1):C51-C55.

33. Wanebo HJ, Schlessinger I, Tashima CK. Severe hypoglycemia associated with terminal lymphomas. Report of five cases. Cancer. 1966;19(10):1451-1458.

34. Marks LJ, Steinke J, Podolsky S, Egdahl RH. Hypoglycemia associated with neoplasia. Ann N Y Acad Sci. 1974;230:147-160.
35. Elhomsy GC, et al. "Hyper-warburgism," a cause of asymptomatic hypoglycemia with lactic acidosis in a patient with non-Hodgkin's lymphoma. J Clin Endocrinol Metab. 2012;97(12):4311-4316.

36. Bentovim L, Amarilio R, Zelzer E. HIF1 $\alpha$ is a central regulator of collagen hydroxylation and secretion under hypoxia during bone development. Development. 2012;139(23):4473-4483.

37. Pfander D, et al. Deletion of Vhlh in chondrocytes reduces cell proliferation and increases matrix deposition during growth plate development. Development. 2004;131(10):2497-2508.

38. Wu C, et al. Oxygen-sensing PHDs regulate bone homeostasis through the modulation of osteoprotegerin. Genes Dev. 2015;29(8):817-831.

39. Gerber HP, Vu TH, Ryan AM, Kowalski J, Werb Z, Ferrara N. VEGF couples hypertrophic cartilage remodeling, ossification and angiogenesis during endochondral bone formation. Nat Med. 1999;5(6):623-628.

40. Maes C, et al. Impaired angiogenesis and endochondral bone formation in mice lacking the vascular endothelial growth factor isoforms VEGF164 and VEGF188. Mech Dev. 2002;111(1-2):61-73.

41. Maes C, et al. Increased skeletal VEGF enhances beta-catenin activity and results in excessively ossified bones. EMBO J. 2010;29(2):424-441.

42. Wu C, Giaccia AJ, Rankin EB. Osteoblasts: a novel source of erythropoietin. Curr Osteoporos Rep. 2014;12(4):428-432.

43. Maes C, et al. VEGF-independent cell-autonomous functions of HIF-1 $\alpha$ regulating oxygen consumption in fetal cartilage are critical for chondrocyte survival. J Bone Miner Res. 2012;27(3):596-609.

44. Dejaeger M, et al. Integrin-linked kinase regulates bone formation by controlling cytoskeletal organization and modulating BMP and Wnt signaling in osteoprogenitors. JBone Miner Res. 2017;32(10):2087-2102. 